\title{
Interferon receptor-deficient mice are susceptible to eschar-associated rickettsiosis
}

Thomas P. Burke ${ }^{1 *}$, Patrik Engström¹, Cuong J. Tran ${ }^{1,2}$, Dustin R. Glasner ${ }^{2,3}$, Diego A. Espinosa ${ }^{2,4}$, Eva Harris ${ }^{2}$, Matthew D. Welch ${ }^{1 *}$

${ }^{1}$ Department of Molecular and Cell Biology, University of California, Berkeley, Berkeley, CA, USA

${ }^{2}$ Division of Infectious Disease and Vaccinology, School of Public Health, University of California,

Berkeley, Berkeley, CA, USA

${ }^{3}$ Current address: Department of Laboratory Medicine, University of California, San Francisco, San Francisco, CA, USA

${ }^{4}$ Current address: Metagenomi, Emeryville, CA, USA

*email: tburke@berkeley.edu; welch@berkeley.edu 


\section{Abstract}

Rickettsia are arthropod-borne pathogens that cause severe human disease worldwide. The

3 spotted fever group (SFG) pathogen Rickettsia parkeri elicits skin lesion (eschar) formation in humans

4 after tick bite. However, intradermal inoculation of inbred mice with millions of bacteria fails to elicit

5 eschar formation or disseminated disease, hindering investigations into understanding eschar-

6 associated rickettsiosis. Here, we report that intradermal infection of mice deficient for both interferon

7 receptors (Ifnar/-/fngr $r^{-/}$) with $R$. parkeri causes eschar formation, recapitulating the hallmark clinical

8 feature of human disease. Intradermal infection with doses that recapitulate tick infestation caused

9 eschar formation and lethality, including with as few as 10 bacteria. Using this model, we found that the

10 actin-based motility protein Sca2 is required for $R$. parkeri dissemination from the skin to internal organs

11 and for causing lethal disease, and that the abundant $R$. parkeri outer membrane protein OmpB

12 contributes to eschar formation. We also found that immunizing mice with sca2 and ompB mutant $R$.

13 parkeri protects against subsequent rechallenge with wild-type bacteria, revealing live-attenuated

14 vaccine candidates. Thus, interferon receptor-deficient mice are a tractable model to investigate

15 rickettsiosis, bacterial virulence factors, and immunity. Our results suggest that differences in interferon

16 signaling in the skin between mice and humans may explain the discrepancy in susceptibility to SFG

17 Rickettsia. 
Introduction

Obligate cytosolic bacterial pathogens in the family Rickettsiaceae are a diverse group of

arthropod-borne microbes that cause severe human disease worldwide, including spotted fever, scrub

typhus, and typhus ${ }^{1-3}$. Human disease caused by the tick-borne spotted fever group (SFG) pathogen

Rickettsia parkeri is characterized by an eschar at the infection site, generalized rash, headache,

fatigue, and fever ${ }^{4}$. There is no approved vaccine for $R$. parkeri or for the more virulent rickettsial pathogens that can cause fatal or latent disease ${ }^{5}$. Moreover, many critical aspects of disease caused by obligate cytosolic bacterial pathogens, including the mechanisms of virulence and immunity, remain unknown, as there are no SFG pathogens that can be handled under biosafety level 2 (BSL2) conditions with corresponding mouse models that recapitulate key features of human disease $\mathrm{e}^{5-7}$.

$R$. parkeri is genetically similar to the more virulent human pathogens $R$. rickettsii and $R$. conorii $i^{8,9}$, and it can be handled under BSL2 conditions. Moreover, mutants can be generated using transposon mutagenesis ${ }^{10,11}$, and small rodents including mice are natural reservoirs for $R$. parkeri ${ }^{12-15}$.

Thus, a mouse model for $R$. parkeri that recapitulates key features of human infection would greatly enhance investigations into understanding rickettsial disease. However, inbred mice including C57BL/6 and BALB/c develop no or minor skin lesions upon intradermal (i.d.) infection with millions of $R$. parkerí . $\mathrm{C} 3 \mathrm{H} / \mathrm{HEJ}$ mice, which harbor a mutation in the gene encoding Toll-like receptor 4 (TLR4), the receptor for extracellular lipopolysaccharide (LPS), have been proposed as models for R. parkeri, yet they do not develop disseminated disease and only develop minor skin lesions upon i.d. inoculation ${ }^{6}$. C57BL/6 mice have also been proposed as models for $R$. parkeri upon intravenous (i.v.) delivery of $10^{8}$ bacteria $^{16}$. However, this dose is substantially higher than the number of $R$. parkeri found in tick saliva or tick salivary glands ${ }^{17}$, and considerable effort is required to generate and concentrate this number of bacteria. An improved mouse model to investigate $R$. parkeri would greatly increase the ability to investigate virulence mechanisms, the host response to infection, and human rickettsial disease. 
restricts viral replication, whereas IFN- $\gamma$ generally restricts intracellular bacterial pathogens ${ }^{18-20}$. We

observed that mice lacking either gene encoding the receptors for IFN-I (Ifnar) or IFN- $\gamma$ (Ifngr) are

resistant to i.v. infection with $R$. parkeri, whereas double mutant $/$ fnar $r^{-/} /$fngr $^{-/-}$mice succumb ${ }^{21}$. This

demonstrates that IFNs redundantly protect against systemic $R$. parkeri. However, the i.v. infection

route does not recapitulate eschar formation or mimic the natural route of dissemination. Moreover,

Ifnar ${ }^{-/}$Ifngr $r^{-/}$mice are resistant to i.v. infection with $10^{5}$ bacteria, which may exceed the amount delivered

upon tick infestation. Further investigations into whether IFNs redundantly protect against $R$. parkeri in

the skin may improve the mouse model for SFG Rickettsia.

A robust mouse model would allow for more detailed investigations into rickettsial virulence

factors. One virulence mechanism shared by divergent cytosolic bacterial pathogens including

Rickettsia, Listeria, Burkholderia, Mycobacterium, and Shigella species, is the ability to undergo actin-

based motility, which facilitates cell to cell spread ${ }^{22,23}$. However, the pathogenic role for many actin-

based motility factors in vivo remains poorly understood. $R$. parkeri actin-based motility differs from that

of other pathogens in that it occurs in two phases, one that requires the RickA protein ${ }^{10,24}$ and the other

that requires the Sca2 protein ${ }^{10,25}$. Only Sca2 is required for efficient cell to cell spread, although it is not required for replication in epithelial cells or for avoiding antimicrobial autophagy ${ }^{10,25-27}$. sca2 mutant

$R$. rickettsii elicit reduced fever in guinea pigs as compared with wild-type (WT) $R$. rickettsii ${ }^{25}$, yet the explanation for reduced fever and the pathogenic role for Sca2 in vivo remains unclear. Additionally,

Sca2 is not essential for dissemination of $R$. parkeri within ticks ${ }^{28}$. A second virulence strategy employed

by intracellular pathogens is the ability to avoid autophagy, which for $R$. parkeri requires outer membrane protein $\mathrm{B}(\mathrm{OmpB})^{27}$. OmpB is important for $R$. parkeri colonization of internal organs in WT mice and for causing lethal disease in IFN receptor-deficient mice after i.v. infection ${ }^{21,27}$; however, the role for OmpB in R. parkeri pathogenesis remains unknown upon i.d. infection. Therefore, unresolved questions remain regarding how Sca2 and OmpB enhance rickettsial pathogenesis.

Here, we use IFN receptor-deficient mice to examine the effects of i.d. inoculation of $R$. parkeri, mimicking the natural route of infection. We observe skin lesions that appear similar to human eschars, 
bioRxiv preprint doi: https://doi.org/10.1101/2020.09.23.310409; this version posted November 11,2020 . The copyright holder for this preprint (which was not certified by peer review) is the author/funder, who has granted bioRxiv a license to display the preprint in perpetuity. It is made available under aCC-BY-NC-ND 4.0 International license.

72 promotes dissemination and is required for causing lethality, and that OmpB contributes to eschar

73 formation. Finally, we demonstrate that immunization with sca2 or ompB mutant $R$. parkeri protects IFN

74 receptor-deficient mice against subsequent challenge with WT bacteria, revealing live-attenuated

75 vaccine candidates. Our study establishes a mouse model to investigate numerous aspects of

76 Rickettsia pathogenesis, including eschar formation, virulence factors, and immunity. More broadly, this

77 work also reveals that a potent, redundant IFN response protects mice from eschar-associated

78 rickettsiosis. 
Results

\section{I.d. infection of Ifnar/-Ifngr//- mice causes lethal disease and skin lesions that are grossly similar}

to human eschars.

Although i.v. delivery can recapitulate an immediate systemic disease for many pathogens, it

does not mimic the natural route of infection for tick-borne pathogens. In contrast, i.d. delivery better

mimics the natural route of infection and allows for investigations into dissemination from the initial

infection site to internal organs. We therefore sought to develop an i.d. murine infection model to better

recapitulate the natural route of tick-borne R. parkeri infection. WT, TIr4 ${ }^{-/}$, Ifnar ${ }^{-/}$, Ifngr ${ }^{-/}$, and Ifnar $r^{-/}$Ifngr

${ }^{\text {/- }}$ C57BL/6J mice, as well as outbred CD-1 mice, were infected i.d. with $10^{7}$ WT R. parkeri and monitored

over time. No or minor dermal lesions appeared at the site of infection in WT, TIr4 ${ }^{-/-}$, Ifnar ${ }^{-/}$, or Ifngr ${ }^{-/-}$

C57BL/6J mice or CD-1 mice (Fig. 1a, Fig. S1a). In contrast, double mutant Ifnar ${ }^{-/ /} /$fngr $^{-/}$C57BL/6J

mice developed large necrotic skin lesions (Fig. 1b) that appeared grossly similar to human eschars

(Fig. 1c). In some cases, tails of $/$ fnar ${ }^{-/} / \mathrm{fngr}^{-/}$or Ifngr $r^{--}$mutant mice became inflamed after i.d. or i.v.

infection (Fig. S1b). These findings demonstrate that interferons redundantly control disease caused

by $R$. parkeri in the skin and that i.d. infection of Ifnar/-/fngr $r^{-/}$mice recapitulates the hallmark manifestation of human disease caused by R. parkeri.

Our previous observations using the i.v. route revealed dose-dependent lethality in Ifnar ${ }^{-/} /$fngr $^{-/-}$

mice, with $10^{7}$ R. parkeri eliciting $100 \%$ lethality and $10^{5}$ R. parkeri eliciting no lethality ${ }^{21}$. R. parkeri are

present in tick saliva at a concentration of approximately $10^{4}$ per $1 \mu$, and approximately $10^{7} R$. parkeri

are found in tick salivary glands ${ }^{17}$. However, the number of bacteria delivered from tick infestation likely varies depending on many factors, and we therefore sought to examine the effects of different doses of

R. parkeri upon i.d. infection of $/$ fnar $^{-/} / \mathrm{fngr}^{-/-}$mice. We observed skin lesion formation at all infectious

doses, from $10^{7}$ to 10 bacteria (Fig. 1d), suggesting that i.d. infection of Ifnar ${ }^{-/} /$fngr ${ }^{-/}$mice elicits lesions with doses similar to what is delivered by tick infestation.

We next sought to quantitatively evaluate the effects of i.d. infection by monitoring animal weight,

104 body temperature, the degree of lesion formation, and lethality. Intradermally-infected Ifnar $r^{-/} /$fngr $^{-/-}$mice $^{-}$

105 lost significant body weight (Fig. 2a; Fig. S2a) and body temperature (Fig. 2b; animals were euthanized 
when body temperature fell below $90^{\circ} \mathrm{F} / 32.2^{\circ} \mathrm{C}$ ) as compared with WT mice, whereas infected TIr4 ${ }^{-/-}$

107 , Ifnar ${ }^{-/}$, Ifngr $r^{-/}$mice did not. To evaluate lesion severity, we scored lesions upon infection with different doses of $R$. parkeri. Whereas $10^{7}$ bacteria elicited similar responses as $10^{5}, 10^{4}, 10^{3}$, and $10^{2}$ bacteria

(Fig. 2c), lesions were less severe when mice were infected with $10^{1}$ bacteria compared with $10^{7}$

bacteria. If mice survived, lesions healed over the course of approximately 15-40 days post infection (d.p.i.) at all doses (Fig. S2b).

To investigate whether i.d. infection by R. parkeri caused lethal disease, we monitored mouse survival over time. Upon i.d. delivery of $10^{7}$ R. parkeri, 8 of 12 Ifnar/-/fngr $r^{-/-}$mice exhibited lethargy, paralysis, or body temperatures below $90^{\circ} \mathrm{F}$, at which point they were euthanized, whereas delivery of the same dose of bacteria to WT and single mutant mice did not elicit lesions and all survived (Fig. 2d). Lower doses of R. parkeri also elicited body weight loss (Fig. 2a), body temperature loss (Fig. S2c), and lethal disease (Fig. 2d) in $/$ fnar $^{-/} /$fngr $^{-/-}$mice. The cause of lethality in this model remains unclear and will require further investigation. Nevertheless, these findings reveal that i.d. infection can cause lethal disease in Ifnar ${ }^{-/}$Ifngr ${ }^{-/}$mice with $~ 10,000$-fold lower dose of bacteria than i.v. infection.

It remained unclear whether i.d. infection could also be used to model dissemination from the skin to internal organs. We therefore evaluated bacterial burdens in spleens and livers of WT and Ifnar

$122{ }^{-}$-Ifngr ${ }^{-/}$mice at 5 d.p.i. by measuring $R$. parkeri plaque-forming units (p.f.u.). Bacteria were not recoverable from spleens and livers of intradermally-infected WT mice, suggesting that they did not disseminate from the skin to internal organs in high numbers (Fig. 2e). In contrast, bacteria were demonstrates that i.d. infection of $/$ fnar/-/fngr/-- mice with $R$. parkeri causes systemic infection and can be used as a model for dissemination from the skin to internal organs.

Ifnar/-Ifngr/- mice do not succumb to intradermal infection with sca2 mutant $R$. parkeri. 
133 (Fig. 3b), we observed that sca2::Tn mutant R. parkeri caused reduced lethality compared to WT

134 bacteria. Similarly, i.d. infection with sca2::Tn mutant bacteria elicited significantly less lethality (Fig.

135 3c) and weight loss (Fig. 3d) as compared to WT bacteria and no severe temperature loss (Fig. S3a).

136 Although we sought to evaluate infection using a sca2 complement strain of $R$. parkeri, our attempts to

137 generate such a strain were unsuccessful. As an alternative strategy, we examined whether the

138 transposon insertion itself had an effect on $R$. parkeri survival in vivo. We evaluated infection of an $R$.

139 parkeri strain that harbors a transposon insertion in MC1_RS08740 (previously annotated as

140 MC1_05535), which has no known role in virulence ${ }^{27}$. I.v. infection with MC1_RS08740::Tn R. parkeri

141 caused lethality to a similar degree as WT R. parkeri (Fig. 3a), demonstrating that the transposon likely

142 does not significantly impact $R$. parkeri fitness in vivo. Together, these findings suggest that the actin-

143 based motility factor Sca2 is required for causing lethal disease in Ifnar ${ }^{-/ /}$Ingr $^{-/-}$mice.

Ifnar/-Ifngr/- mice exhibit similar skin lesion formation and vascular damage upon i.d. infection

with WT and sca2::Tn R. parkeri.

We next examined whether Sca2 facilitates $R$. parkeri dissemination throughout the skin and whether Sca2 is required for lesion formation. Unexpectedly, upon i.d. inoculation, Ifnar ${ }^{--}$Ifngr ${ }^{--}$mice infected with sca2::Tn mutant bacteria developed skin lesions that were of similar severity to lesions caused by WT R. parkeri; however, the lesions elicited by sca 2 mutant bacteria appeared significantly earlier than lesions caused by WT bacteria (Fig. 3e). Further examinations will be required to better evaluate this observation; however, it may suggest that actin-based motility enables $R$. parkeri to avoid a rapid onset of inflammation in the skin. To evaluate $R$. parkeri dissemination within the skin, we used a fluorescence-based assay that measures vascular damage as a proxy for pathogen dissemination ${ }^{29}$. 
infection (Fig. S4a). Similar results were observed upon infection with $10^{6}$ or $10^{5}$ bacteria (Fig. S4b,c).

However, significantly more fluorescence was observed in the skin of infected Ifnar ${ }^{-/}$Ifngr ${ }^{-/}$mice as

compared to WT mice (Fig. $\mathbf{3 g}$ ), demonstrating that interferons protect against increased vascular

during i.d. infection of Ifnar ${ }^{/-1}$ fng $r^{/-}$mice.

\section{R. parkeri Sca2 promotes dissemination from the skin to spleens and livers.}

Among the factors that mediate actin-based motility, the L. monocytogenes actin-based motility

factor ActA is one of the best understood. ActA enables L. monocytogenes to spread from cell to cell ${ }^{22,23}$,

escape antimicrobial autophagy ${ }^{30-33}$, proliferate in mouse organs after i.v. infection ${ }^{34,35}$, and cause lethal

disease in mice ${ }^{36,37}$. We initially hypothesized that $R$. parkeri Sca2 plays a similar pathogenic role in

vivo to ActA, which we found is required for bacterial survival in spleens and livers upon i.v. delivery

(Fig. 3h), in agreement with previous experiments ${ }^{34,35}$. However, when we examined bacterial burdens

upon i.v. infection of Ifnar/-Ifngr/- mice with $R$. parkeri, similar amounts of WT and sca2::Tn bacteria

were recovered in spleens (Fig. 3i). We were also surprised to find that significantly more sca2::Tn than

WT R. parkeri were recovered in livers (Fig. 3i). The explanation for higher sca2::Tn burdens in livers

remains unclear. Nevertheless, these data reveal that Sca2 is likely not essential for $R$. parkeri survival in blood, invasion of host cells, or intracellular survival in spleens and livers.

We next evaluated the role for Sca2 in $R$. parkeri dissemination by measuring p.f.u. in spleens

and livers following i.d. infection of Ifnar $r^{-/} / \mathrm{fng} r^{-/-}$mice. After i.d. infection, sca2::Tn mutant bacteria were

$\sim 20$-fold reduced in their abundance in spleens and $\sim 2$-fold reduced in their abundance in livers as 
Sca2-mediated actin-based motility is required for efficient plaque formation and cell to cell spread by $R$. parkeri in vitro ${ }^{10,25}$. However, it remains unclear if Sca2 enables $R$. parkeri to escape detection or restriction by innate immunity. The actin-based motility factor ActA enables $L$. monocytogenes to avoid autophagy ${ }^{32,33}$, and the antimicrobial guanylate binding proteins (GBPs) inhibit Shigella flexneri actin-based motility ${ }^{38}$. We therefore sought to evaluate whether Sca2-mediated actinbased motility enables $R$. parkeri to evade innate immunity in vitro. We found that the sca2::Tn mutant cells $^{10,25}$. We also examined whether Sca2 contributed to R. parkeri survival or growth in bone marrowderived macrophages (BMDMs), which can restrict other $R$. parkeri mutants that grow normally in endothelial cells ${ }^{27}$. However, no significant difference in bacterial survival was observed between WT and sca2::Tn bacteria in BMDMs in the presence or absence of IFN- $\beta$ (Fig. S5b). WT and sca2 mutant Moreover, we found that the anti-rickettsial factor GBP2 localized to the surface of sca2::Tn mutant $R$. innate immunity in vitro.

Ifnar-Ifngr $r^{-/-}$mice exhibit less severe skin lesions upon infection with a highly attenuated $R$.

remained unclear whether skin lesion formation in $I f n a r^{-/} / f n g r^{--}$mice was influenced by bacterial both a transposon and a stop codon in $o m p B^{27}$, and is severely attenuated in vivo ${ }^{21,27}$. In contrast with 210 WT bacteria, i.d. infection of Ifnar/-/fngr $r^{-/}$mice with ompB::Tn ${ }^{\mathrm{STOP}}$ R. parkeri caused no lethality (Fig. 2114 a) or reduced weight loss (Fig. 4b). The ompB::Tn ${ }^{\text {STOP }}$ mutant $R$. parkeri also caused significantly less 212 severe skin lesions than WT bacteria (Fig. 4c). These findings suggest that Ifnar ${ }^{-/} /$fngr $r^{-/}$mice can be used as a model to identify bacterial genes important for eschar formation. 
Immunizing Ifnar/-Ifngr//- mice with attenuated $R$. parkeri mutants protects against subsequent

rechallenge.

There is currently no available vaccine to protect against SFG Rickettsia, which can cause

severe and lethal human disease ${ }^{5,39}$, and investigations into identifying live attenuated vaccine

immunization with attenuated $R$. parkeri mutants would protect against subsequent re-challenge with a

R. parkeri and $40 \mathrm{~d}$ later were intravenously re-challenged with $10^{7}$ WT $R$. parkeri, which is approximately 10 -times a $50 \%$ lethal dose $\left(L_{50}\right)^{21}$. All mice immunized with sca2 or ompB mutant $R$.

immunized with ompB and sca2 mutants also did not lose significant weight (Fig. 5b) or body

temperature (Fig. 5c). These data indicate that attenuated $R$. parkeri mutants elicit a robust protective immune response, and that Ifnar ${ }^{-/}$Ifngr $r^{-/}$mice may serve as tools to develop live attenuated $R$. parkeri vaccine candidates.

\section{Discussion}

In this study, we show that IFN-I and IFN- $\gamma$ redundantly protect inbred mice from escharassociated rickettsiosis and disseminated disease by $R$. parkeri. Eschar formation is the hallmark clinical feature of human disease caused by R. parkeri ${ }^{4}$, and thus these findings suggest that the striking difference between human and mouse susceptibilities to $R$. parkeri may be due to IFN signaling in the skin. Using this mouse model, we uncover a role for $R$. parkeri Sca2 in dissemination, for OmpB in skin lesion formation, and for both proteins in causing lethal disease. We further demonstrate that attenuated R. parkeri mutants elicit long-lasting immunity, revealing live attenuated vaccine candidates. Obligate cytosolic bacterial pathogens cause a variety of severe human diseases on six continents ${ }^{1,40}$, and the 
response to infection, the molecular determinants of human disease, and propagation of tick-borne pathogens in wildlife reservoirs.

Our finding that i.d. infection of Ifnar ${ }^{-/} / \mathrm{fng} r^{-/-}$mice causes eschar formation, the hallmark of $R$.

parkeri infection in humans ${ }^{4}$, may indicate that the human IFN response is less well adapted to control broadly, investigating the IFN response in the skin may lead us to better understand diseases caused by other arthropod-borne pathogens. One example may be Orientia tsutsugamushi, the causative agent of scrub typhus ${ }^{41}$, a prevalent but poorly understood tropical disease endemic to Southeast Asia ${ }^{1,42,43}$. burgdorferi, a tick-borne pathogen that causes a skin rash at the site of tick bite as a hallmark feature of Lyme disease ${ }^{44}$, the most prevalent tick-borne disease in the United States ${ }^{44,45}$. Existing mouse models also do not recapitulate skin rash formation following $B$. burgdorferi infection ${ }^{46,47}$. Further investigations into how IFNs protect the skin in mice may therefore reveal aspects of human disease caused by other arthropod-borne pathogens.

Our study further highlights the utility of mouse models that mimic natural routes of infection. Infection via the i.v. and intraperitoneal (i.p.) routes can mimic systemic disease, yet these are unnatural routes for many microbes, including food-borne, arthropod-borne, or aerosol-borne pathogens. Our observation that i.d. infection can cause lethal disease with as few as 10 bacteria, $\sim 10,000$ fewer bacteria than i.v. infection ${ }^{21}$, suggests that $R$. parkeri may be highly adapted to reside in the skin. However, this model could be further improved by investigating the role for tick vector components in pathogenesis. Saliva from ticks, mosquitos, and sand flies enhances pathogenesis of arthropod-borne 
for tick vector components such as tick saliva in R. parkeri pathogenesis. Developing improved murine

infection models that mimic the natural route of infection, including with tick saliva or the tick vector, is

critical to better understand the virulence and transmission of tick-borne pathogens.

reported that i.v. infection of guinea pigs with sca2 mutant $R$. rickettsii did not elicit fever ${ }^{25}$. Our results suggest that Sca2-mediated actin-based motility by Rickettsia may facilitate dissemination in host reservoirs, although we cannot rule out other roles for Sca2 that do not involve actin assembly. $R$.

prowazekii and $R$. typhi, which cause severe human disease, encode a fragmented sca2 gene ${ }^{52}$, and undergo no or dramatically reduced frequency of actin-based motility, respectively ${ }^{53,54}$. Although it remains unclear why some Rickettsia species lost the ability to undergo actin-based motility, Sca2 is dispensable for $R$. parkeri dissemination in the tick vector ${ }^{28}$, suggesting that actin-based motility may play a specific role in dissemination within mammalian hosts.

We find that sca2 or ompB mutant $R$. parkeri elicit a robust protective immune response in Ifnar antibody responses in guinea pigs ${ }^{25}$, and expands upon these findings by demonstrating protection from rechallenge and revealing additional vaccine candidates. There are currently limited vaccine candidates that protect against rickettsial disease ${ }^{5}$. Identifying new vaccine candidates may reveal avenues to protect against tick-borne infections and aerosolized Rickettsia, which are extremely virulent and 292 potential bioterrorism agents ${ }^{55}$, as well as against Brill-Zinsser disease, caused by latent R. prowazekii. 
bioRxiv preprint doi: https://doi.org/10.1101/2020.09.23.310409; this version posted November 11,2020 . The copyright holder for this preprint (which was not certified by peer review) is the author/funder, who has granted bioRxiv a license to display the preprint in perpetuity. It is made available under aCC-BY-NC-ND 4.0 International license.

294 Rickettsia species are warranted to better define the mechanisms of protection. These findings on

295 immunity may also help develop R. parkeri as an antigen delivery platform. R. parkeri resides directly

296 in the host cytosol for days and could potentially be engineered to secrete foreign antigens for

297 presentation by major histocompatibility complex I. In summary, the mouse model described here will

298 facilitate future investigations into numerous aspects of $R$. parkeri infection, including actin-based

299 motility and immunity, and may serve as model for other arthropod-borne pathogens. 
Methods

\section{Bacterial preparations}

when $\sim 90 \%$ of cells were rounded due to infection. Scraped cells were then centrifuged at $12,000 \mathrm{~g}$ for

$20 \mathrm{~min}$ at $4^{\circ} \mathrm{C}$. Pelleted cells were resuspended in K-36 buffer $\left(0.05 \mathrm{M} \mathrm{KH}_{2} \mathrm{PO}_{4}, 0.05 \mathrm{M} \mathrm{K}_{2} \mathrm{HPO}_{4}, 100\right.$

$\mathrm{mM} \mathrm{KCl}, 15 \mathrm{mM} \mathrm{NaCl}, \mathrm{pH} 7$ ) and dounced for $\sim 40$ strokes at $4^{\circ} \mathrm{C}$. The suspension was then centrifuged

at $200 \mathrm{~g}$ for $5 \mathrm{~min}$ at $4^{\circ} \mathrm{C}$ to pellet host cell debris. Supernatant containing $R$. parkeri was overlaid on a 30\% MD-76R (Merry X-Ray) gradient solution in ultracentrifuge tubes (Beckman/Coulter Cat 344058).

313 Gradients were centrifuged at 18,000 r.p.m. in an SW-28 ultracentrifuge swinging bucket rotor

314 (Beckman/Coulter) for $20 \mathrm{~min}$ at $4^{\circ} \mathrm{C}$. These '30\% prep' bacterial pellets were resuspended in brain heart infusion $(\mathrm{BHI})$ media $(\mathrm{BD}, 237500)$, aliquoted, and stored at $-80^{\circ} \mathrm{C}$. Titers were determined by plaque assays by serially diluting the $R$. parkeri in 6-well plates containing confluent Vero cells. Plates were spun for $5 \mathrm{~min}$ at $300 \mathrm{~g}$ in an Eppendorf 5810R centrifuge and at $24 \mathrm{~h}$ post infection (h.p.i.); the upstream stop codon in $o m p B$, as previously described ${ }^{27}$.

\section{Deriving bone marrow macrophages}

326 fibulas were excised. Bones were sterilized with $70 \%$ ethanol and washed with BMDM media (20\% FBS 
327 (HyClone), 0.1\% $\beta$-mercaptoethanol, 1\% sodium pyruvate, 10\% conditioned supernatant from 3 T3

328 fibroblasts, in DMEM (Gibco) with $4.5 \mathrm{gl}^{-1} \mathrm{glucose}$ and $100 \mu \mathrm{g} / \mathrm{ml}$ streptomycin and $100 \mathrm{U} / \mathrm{ml}$ penicillin),

329 and ground with a mortar and pestle. Bone homogenate was passed through a 70 um nylon cell strainer

330 (Thermo Fisher Scientific, 08-771-2) for particulate removal. Filtrates were then centrifuged at $290 \mathrm{~g}$ in

331 an Eppendorf 5810R centrifuge for $8 \mathrm{~min}$, supernatant was aspirated, and the pellet was resuspended

332 in BMDM media. Cells were plated in $30 \mathrm{ml}$ BMDM media in non-TC-treated $15 \mathrm{~cm}$ petri dishes at a

333 ratio of 10 dishes per 2 femurs/tibias and incubated at $37^{\circ} \mathrm{C}$. An additional $30 \mathrm{ml}$ of BMDM media was

334 added $3 \mathrm{~d}$ later. At $7 \mathrm{~d}$ the media was aspirated, $15 \mathrm{ml}$ cold PBS (Gibco, 10010-023) was added, and

335 cells were incubated at $4^{\circ} \mathrm{C}$ with for $10 \mathrm{~min}$. BMDMs were scraped from the plate, collected in a $50 \mathrm{ml}$

336 conical tube, and centrifuged at $290 \mathrm{~g}$ for $5 \mathrm{~min}$. PBS was aspirated, and cells were resuspended in

337 BMDM media with $30 \%$ FBS and $10 \%$ DMSO at $10^{7}$ cells $/ \mathrm{ml}$. $1 \mathrm{ml}$ aliquots were stored at $-80^{\circ} \mathrm{C}$ for 24

$338 \mathrm{~h}$ in Styrofoam boxes and then moved to long-term storage in liquid nitrogen.

\section{Infections in vitro}

HMEC-1 cells (obtained from the UC Berkeley Cell Culture Facility and authenticated by short-

342 tandem-repeat analysis) were passaged 2-3 times weekly and grown at $37^{\circ} \mathrm{C}$ with $5 \% \mathrm{CO}_{2}$ in $\mathrm{DMEM}$

343 containing $10 \mathrm{mM} \mathrm{L-glutamine} \mathrm{(Sigma,} \mathrm{M8537),} \mathrm{supplemented} \mathrm{with} 10 \%$ heat-inactivated FBS

344 (HyClone), $1 \mu \mathrm{g} / \mathrm{mL}$ hydrocortisone (Spectrum Chemical, CO137), $10 \mathrm{ng} / \mathrm{mL}$ epidermal growth factor

345 (Thermo Fisher Scientific, CB40001; Corning cat. no. 354001), and $1.18 \mathrm{mg} / \mathrm{mL}$ sodium bicarbonate.

346 HMEC media was prepared every 1-2 months, and aliquoted and stored at $4^{\circ} \mathrm{C}$. To prepare $\mathrm{HMEC}-1$

347 cells for infection, cells were treated with 0.25\% trypsin-EDTA (Thermo Fisher Scientific); the number

348 of cells was counted using a hemocytometer (Bright-Line), and $3 \times 10^{4}$ cells were plated into 24-well 349 plates $48 \mathrm{~h}$ prior to infection.

350 To plate macrophages for infection, BMDM aliquots were thawed on ice, diluted into $9 \mathrm{ml}$ of

351 DMEM, centrifuged at $290 \mathrm{~g}$ for $5 \mathrm{~min}$ in an Eppendorf $5810 \mathrm{R}$ centrifuge, and the pellet was resuspended

352 in $10 \mathrm{ml}$ BMDM media without antibiotics. $5 \times 10^{5}$ cells were plated into 24 -well plates. Approximately

$35316 \mathrm{~h}$ later, "30\% prep" R. parkeri were thawed on ice and diluted into fresh BMDM media to either $10^{6}$ 
354 p.f.u. $/ \mathrm{ml}$ or $2 \times 10^{5}$ p.f.u./ml. Media was then aspirated from the BMDMs, replaced with $500 \mu \mathrm{l}$ media

355 containing $R$. parkeri, and plates were spun at $300 \mathrm{~g}$ for $5 \mathrm{~min}$ in an Eppendorf $5810 \mathrm{R}$ centrifuge.

356 Infected cells were incubated in a humidified CEDCO 1600 incubator set to $33^{\circ} \mathrm{C}$ and $5 \% \mathrm{CO}_{2}$.

357 Recombinant mouse IFN- $\beta$ (PBL, 12405-1) was added directly to infected cells after spinfection.

For measuring p.f.u., supernatants from infected BMDMs were aspirated, and each well was

washed twice with $500 \mu \mathrm{l}$ sterile milli-Q-grade water. After adding $1 \mathrm{ml}$ of sterile milli-Q water to each

well, macrophages were lysed by repeated pipetting. Serial dilutions of lysates were added to confluent

Vero cells in 12 well plates. Plates were spun at $300 \mathrm{~g}$ using an Eppendorf $5810 \mathrm{R}$ centrifuge for 5 min

at room temperature and incubated at $33^{\circ} \mathrm{C}$ overnight. At $\sim 16$ h.p.i., media was aspirated and replaced

with $2 \mathrm{ml} /$ well of DMEM containing $0.7 \%$ agarose and 5\% FBS (GemCell). At $\sim 6$ d.p.i., $1 \mathrm{ml}$ of DMEM containing $0.7 \%$ agarose, 1\% FBS (GemCell), $200 \mu \mathrm{g} / \mathrm{ml}$ amphotericin B (Invitrogen, 15290-018), and

$2.5 \%$ neutral red (Sigma) was added to each well. Plaques were then counted after $24 \mathrm{~h}$.

Microscopy, LDH, and IFN-I experiments were performed as described ${ }^{21}$.

\section{Animal experiments}

Animal research was conducted under a protocol approved by the University of California, performed in a biosafety level 2 facility. All animals were maintained at the University of California, were between 8 and 20 weeks old at the time of initial infection. Mice were selected for experiments based on their availability, regardless of sex. The sex of mice used for survival after i.d. infection and 
availability of mice and the capacity to process or measure samples within a given time. After the first

experiment, a Power Analysis was conducted to determine subsequent group sizes. All mice were of

the C57BL/6J background, except for outbred CD-1 mice. All mice were healthy at the time of infection

and were housed in microisolator cages and provided chow, water, and bedding. No mice were

administered antibiotics or maintained on water with antibiotics. Experimental groups were littermates

of the same sex that were randomly assigned to experimental groups. For experiments with mice

deficient in Ifnar and Ifngr, mice were immediately euthanized if they exhibited severe degree of

infection, as defined by a core body temperature dropping below $90^{\circ} \mathrm{F}$ or lethargy that prevented normal

movement.

\section{Mouse genotyping}

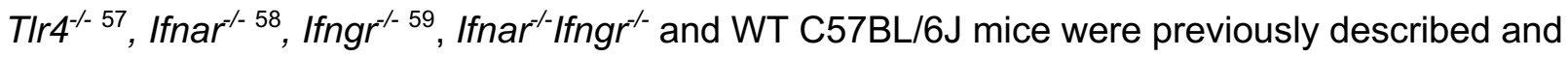

originally obtained from Jackson Laboratories. CD-1 mice were obtained from Charles River. For genotyping, ear clips were boiled for 15 min in $60 \mu$ of $25 \mathrm{mM} \mathrm{NaOH}$, quenched with $10 \mu \mathrm{l}$ Tris- $\mathrm{HCl} \mathrm{pH}$

5.5, and $2 \mu \mathrm{l}$ of lysate was used for PCR using SapphireAMP (Takara, RR350) and gene-specific primers. Primers used were: Ifnar forward (F): CAACATACTACAACGACCAAGTGTG; Ifnar WT reverse (R): AACAAACCCCCAAACCCCAG; Ifnar ${ }^{\prime-~ R: ~ A T C T G G A C G A A G A G C A T C A G G ; ~ I f n g r ~(F): ~}$

CTCGTGCTTTACGGTATCGC; $\quad$ Ifngr $\quad(\mathrm{R}): \quad$ TCGCTTTCCAGCTGATGTACT; $\quad$ WT $\quad$ TIr4 $\quad(\mathrm{F})$ :

and $T / r 4^{--}(R):$ TGTTGGGTCGTTTGTTCGGATCCGTCG.

403

\section{Mouse infections}

For mouse infections, $R$. parkeri was prepared by diluting 30\%-prep bacteria into cold sterile PBS on ice. Bacterial suspensions were kept on ice during injections. For i.d. infections, mice were anaesthetized with $2.5 \%$ isoflurane via inhalation. The right flank of each mouse was shaved with a hair trimmer (Braintree CLP-41590), wiped with $70 \%$ ethanol, and $50 \mu$ l of bacterial suspension in PBS was 
injected intradermally using a 30.5-gauge needle. Mice were monitored for $\sim 3$ min until they were fully

awake. No adverse effects were recorded from anesthesia. For i.v. infections, mice were exposed to a

heat lamp while in their cages for approximately $5 \mathrm{~min}$ and then each mouse was moved to a mouse

restrainer (Braintree, TB-150 STD). The tail was sterilized with $70 \%$ ethanol, and $200 \mu$ of bacterial

For fluorescent dextran experiments, mice were intravenously injected with $150 \mu \mathrm{l}$ of $10 \mathrm{kDa}$

dextran conjugated with Alexa Fluor 680 (D34680; Thermo Fisher Scientific) at a concentration of 1

$\mathrm{mg} / \mathrm{ml}$ in sterile $\mathrm{PBS}^{29}$. As a negative control, mice with no $R$. parkeri infection were injected with

fluorescent dextran. As an additional negative control, uninfected mice were injected intravenously with

PBS instead of fluorescent dextran. At $2 \mathrm{~h}$ post-injection, mice were euthanized with $\mathrm{CO}_{2}$ and cervical

dislocation, doused with $70 \%$ ethanol, and skin surrounding the injection site (approximately $2 \mathrm{~cm}$ in each direction) was removed. Connective tissue between the skin and peritoneum was removed, and skin was placed hair-side-up on a $15 \mathrm{~cm}$ Petri dish. Skin was imaged with an LI-COR Odyssey CLx (LICOR Biosciences), and fluorescence was quantified using ImageStudioLite v5.2.5. The skin from mice with no injected fluorescent dextran was used as the background measurement. Skin from mice injected with fluorescent dextran but no R. parkeri was normalized to an arbitrary number (100), and R. parkeriinfected samples were normalized to this value (R. parkeri-infected / uninfected X 100). The number of pixels at the injection site area was maintained across experiments $(7,800$ for small area and 80,000 for the large area).

All mice in this study were monitored daily for clinical signs of disease throughout the course of infection, such as hunched posture, lethargy, scruffed fur, paralysis, facial edema, and lesions on the skin of the flank and tail. If any such manifestations were observed, mice were monitored for changes

431 in body weight and temperature. If a mouse displayed severe signs of infection, as defined by a 432 reduction in body temperature below $90^{\circ} \mathrm{F}$ or an inability to move normally, the animal was immediately 433 and humanely euthanized using $\mathrm{CO}_{2}$ followed by cervical dislocation, according to IACUC-approved 434 procedures. Pictures of skin and tail lesions were obtained with permission from the Animal Care and 
Use Committee Chair and the Office of Laboratory and Animal Care. Pictures were captured with an

Apple iPhone 8, software v13.3.1.

For harvesting spleens and livers, mice were euthanized at the indicated pre-determined times

and doused with ethanol. Mouse organs were extracted and deposited into $50 \mathrm{ml}$ conical tubes

were homogenized for $\sim 10$ s using an immersion homogenizer (Fisher, Polytron PT 2500E) at $\sim 22,000$

r.p.m. Organ homogenates were spun at $290 \mathrm{~g}$ for $5 \mathrm{~min}$ to pellet the cell debris (Eppendorf $5810 \mathrm{R}$

centrifuge). $20 \mu$ l of organ homogenates were then serial diluted into 12-well plates containing confluent

Vero cells. The plates were then spun at $260 \mathrm{~g}$ for $5 \mathrm{~min}$ at room temperature (Eppendorf $5810 \mathrm{R}$

centrifuge) and incubated at $33^{\circ} \mathrm{C}$. To reduce the possibility of contamination, organ homogenates were

plated in duplicate and the second replicate was treated with $50 \mu \mathrm{g} / \mathrm{ml}$ carbenicillin (Sigma) and 200

$\mu \mathrm{g} / \mathrm{ml}$ amphotericin B (Gibco). The next day, at approximately 16 h.p.i., the cells were gently washed

by replacing the existing media with $1 \mathrm{ml}$ DMEM containing 2\% FBS (GemCell). The media were then

aspirated and replaced with $2 \mathrm{ml} /$ well of DMEM containing $0.7 \%$ agarose, $5 \%$ FBS, and $200 \mu \mathrm{g} / \mathrm{ml}$

amphotericin B. When plaques were visible at 6 d.p.i., $1 \mathrm{ml}$ of DMEM containing $0.7 \%$ agarose, $1 \%$

FBS, and 2.5\% neutral red (Sigma) was added to each well, and plaques were counted at 24 h.p.i.

\section{Statistical analysis}

Statistical parameters and significance are reported in the figure legends. For comparing two

sets of data, a two-tailed Student's T test was performed. For comparing two sets of in vivo p.f.u. data,

Mann-Whitney $U$ tests were used. For comparing two survival curves, log-rank (Mantel-Cox) tests were

used. For comparing curves of two samples (mouse health, weight, and temperature), two-way

ANOVAs were used. For two-way ANOVAs, if a mouse was euthanized prior to the statistical endpoint,

the final value that was recorded for the mouse was repeated until the statistical endpoint. For two-way 
controls. Error bars indicate standard deviation (SD) for in vitro experiments and standard error of the mean (SEM) for in vivo experiments. All other graphical representations are described in the figure legends. Statistical analyses were performed using GraphPad PRISM v7.0.

\section{Data availability}

WT and ompB mutant $R$. parkeri were authenticated by whole genome sequencing and are

available in the NCBI Trace and Short-Read Archive; Sequence Read Archive (SRA), accession

numbers: SRX4401164 (WT) and SRX4401167 (ompB::Tn ${ }^{\text {STOP}) . ~ R a w ~ D a t a ~ f o r ~ f i g u r e s ~ i n ~ t h e ~ m a i n ~ t e x t ~}$

are available in Supplemental Table 1.

471

\section{Competing interests}

The authors declare no competing interests.

\section{Author contributions}

T.P.B. performed and analyzed experiments. C.J.T., P.E., D.R.G., and D.A.E. contributed to performing experiments and provided reagents. T.P.B. wrote the original draft of this manuscript with guidance from M.D.W. Critical reading and edits of the manuscript were provided by C.J.T., P.E., and M.D.W. Supervision was provided by T.P.B. and M.D.W.

\section{Acknowledgements}

We thank Neil Fisher for editing this manuscript. P.E. was supported by postdoctoral fellowships

483 from the Sweden-America Foundation. M.D.W. was supported by NIH/NIAID grants R01Al109044 and 484 R21Al138550. D.R.G., D.A.E., and E.H. were partially supported by NIH/NIAID grant R01 Al24493 485 (E.H.). 


\section{Bibliography}

487 1. Bonell, A., Lubell, Y., Newton, P. N., Crump, J. A. \& Paris, D. H. Estimating the burden of scrub typhus: A systematic review. PLoS Negl. Trop. Dis. 11, e0005838 (2017).

489

2. Fang, R., Blanton, L. S. \& Walker, D. H. Rickettsiae as Emerging Infectious Agents. Clinics in Laboratory Medicine vol. 37 383-400 (2017).

491

3. Sahni, A., Fang, R., Sahni, S. K. \& Walker, D. H. Pathogenesis of Rickettsial Diseases: Pathogenic and Immune Mechanisms of an Endotheliotropic Infection. Annu. Rev. Pathol. Mech. Dis. 14, 127-152 (2019).

4. Paddock, C. D. et al. Rickettsia parkeri Rickettsiosis and Its Clinical Distinction from Rocky Mountain Spotted Fever . Clin. Infect. Dis. 47, 1188-1196 (2008).

5. Osterloh, A. Immune response against rickettsiae: lessons from murine infection models. Med. Microbiol. Immunol. 206, 403-417 (2017).

6. Grasperge, B. J. et al. Susceptibility of inbred mice to Rickettsia parkeri. Infect. Immun. 80, 1846-1852 (2012).

500 7. Sunyakumthorn, P. et al. An Intradermal Inoculation Model of Scrub Typhus in Swiss CD-1

501 Mice Demonstrates More Rapid Dissemination of Virulent Strains of Orientia tsutsugamushi. PLoS One 8, (2013).

8. Roux, V. \& Raoult, D. Phylogenetic analysis of members of the genus Rickettsia using the gene encoding the outer-membrane protein rOmpB (ompB). Int. J. Syst. Evol. Microbiol. 50, 1449-

9. Goddard, J. Historical and recent evidence for close relationships among Rickettsia parkeri, R. conorii, R. africae, and R. sibirica: Implications for rickettsial taxonomy. J. Vector Ecol. 34, 238242 (2009).

10. Reed, S. C. O., Lamason, R. L., Risca, V. I., Abernathy, E. \& Welch, M. D. Rickettsia actinbased motility occurs in distinct phases mediated by different actin nucleators. Curr. Biol. 24, 
of Rickettsia parkeri yields numerous mutations that impact infection. PLoS One 13, 1-12 (2018).

12. Moraru, G. M. et al. Evidence of antibodies to spotted fever group rickettsiae in small mammals and quail from Mississippi. Vector-Borne Zoonotic Dis. 13, 1-5 (2013). cotton rats and bobwhite quail with Rickettsia parkeri. Parasites and Vectors 6, 1-5 (2013).

14. Krawczak, F. S. et al. Ecology of a tick-borne spotted fever in southern Brazil. Exp. Appl. Acarol. 70, 219-229 (2016).

15. Barbieri, A. R. M. et al. Species richness and seasonal dynamics of ticks with notes on rickettsial infection in a Natural Park of the Cerrado biome in Brazil. Ticks Tick. Borne. Dis. 10,

16. Londoño, A. F., Mendell, N. L., Walker, D. H. \& Bouyer, D. H. A biosafety level-2 dose-

17. Suwanbongkot, C. et al. Spotted fever group rickettsia infection and transmission dynamics in Amblyomma maculatum. Infect. Immun. 87, e00804-18 (2019).

18. Raniga, K. \& Liang, C. Interferons: Reprogramming the metabolic network against viral infection. Viruses 10, 1-21 (2018).

19. Billiau, A. \& Matthys, P. Interferon-y: A historical perspective. Cytokine and Growth Factor Reviews vol. 20 97-113 (2009).

20. Meunier, E. \& Broz, P. Interferon-inducible GTPases in cell autonomous and innate immunity. Cell. Microbiol. 18, 168-180 (2016). pathogenesis. Nat. Microbiol. 5, 688-696 (2020).

537 22. Choe, J. E. \& Welch, M. D. Actin-based motility of bacterial pathogens: mechanistic diversity and its impact on virulence. Pathog. Dis. 74, ftw099 (2016).

539 23. Lamason, R. L. \& Welch, M. D. Actin-based motility and cell-to-cell spread of bacterial 
pathogens. Current Opinion in Microbiology vol. 35 48-57 (2017).

541 24. Jeng, R. L. et al. A Rickettsia WASP-like protein activates the Arp2/3 complex and mediates actin-based motility. Cell. Microbiol. 6, 761-769 (2004).

543 25. Kleba, B., Clark, T. R., Lutter, E. I., Ellison, D. W. \& Hackstadt, T. Disruption of the Rickettsia rickettsii Sca2 autotransporter inhibits actin-based motility. Infect. Immun. 78, 2240-2247 (2010).

26. Haglund, C. M., Choe, J. E., Skau, C. T., Kovar, D. R. \& Welch, M. D. Rickettsia Sca2 is a

27. Engström, P. et al. Evasion of autophagy mediated by Rickettsia surface protein OmpB is critical for virulence. Nat. Microbiol. 4, 2538-2551 (2019).

28. Harris, E. K. et al. Role of Sca2 and RickA in the dissemination of Rickettsia parkeri in Amblyomma maculatum. Infect. Immun. 86, e00123-18 (2018).

29. Glasner, D. R. et al. Dengue virus NS1 cytokine-independent vascular leak is dependent on endothelial glycocalyx components. PLoS Pathog. 13, 1-22 (2017).

30. Cheng, M. I., Chen, C., Engström, P., Portnoy, D. A. \& Mitchell, G. Actin-based motility allows Listeria monocytogenes to avoid autophagy in the macrophage cytosol. Cell. Microbiol. 20,

31. Mitchell, G. et al. Listeria monocytogenes triggers noncanonical autophagy upon phagocytosis, but avoids subsequent growth-restricting xenophagy. Proc. Natl. Acad. Sci. U. S. A. 115, E210E217 (2017).

32. Yoshikawa, Y. et al. Listeria monocytogenes ActA-mediated escape from autophagic recognition. Nat. Cell Biol. 11, 1233-1240 (2009). ActA is a key player in evading autophagic recognition. Autophagy 5, 1220-1221 (2009). 
35. Le Monnier, A. et al. ActA is required for crossing of the fetoplacental barrier by Listeria monocytogenes. Infect. Immun. 75, 950-957 (2007).

36. Goossens, P. L., Milon, G. \& Bevan, M. Induction of protective CD8+ T lymphocytes by an attenuated listeria monocytogenes actA mutant. Int. Immunol. 4, 1413-1418 (1992).

37. Brundage, R. A., Smith, G. A., Camilli, A., Theriot, J. A. \& Portnoy, D. A. Expression and phosphorylation of the Listeria monocytogenes actA protein in mammalian cells. Proc. Natl. Acad. Sci. U. S. A. 90, 11890-11894 (1993).

38. Piro, A. S. et al. Detection of cytosolic shigella flexneri via a C-terminal triple-arginine motif of GBP1 inhibits actin-based motility. MBio 8, e01979-17 (2017).

39. Dantas-Torres, F. Rocky Mountain spotted fever. Lancet Infectious Diseases vol. 7 724-732 (2007).

40. Abdad, M. Y., Abdallah, R. A., Fournier, P. E., Stenos, J. \& Vasoo, S. A concise review of the epidemiology and diagnostics of rickettsioses: Rickettsia and orientia spp. Journal of Clinical Microbiology vol. 56 e01728-17 (2018).

41. Rajapakse, S., Weeratunga, P., Sivayoganathan, S. \& Fernando, S. D. Clinical manifestations of scrub typhus. Transactions of the Royal Society of Tropical Medicine and Hygiene vol. 111 43-54 (2017).

42. Kelly, D. J., Foley, D. H. \& Richards, A. L. A Spatiotemporal Database to Track Human Scrub Typhus Using the VectorMap Application. PLoS Neglected Tropical Diseases vol. 9 e0004161 (2015).

43. Richards, A. L. \& Jiang, J. Scrub typhus: Historic perspective and current status of the worldwide presence of Orientia species. Tropical Medicine and Infectious Disease vol. 549 (2020).

44. Bratton, R. L., Whiteside, J. W., Hovan, M. J., Engle, R. L. \& Edwards, F. D. Diagnosis and treatment of lyme disease. Mayo Clinic Proceedings vol. 83 566-571 (2008).

45. Schwartz, A. M., Hinckley, A. F., Mead, P. S., Hook, S. A. \& Kugeler, K. J. Surveillance for lyme disease - United States, 2008-2015. MMWR Surveill. Summ. 66, (2017). 
594 46. Barthold, S. W., Beck, D. S., Hansen, G. M., Terwilliger, G. A. \& Moody, K. D. Lyme borreliosis in selected strains and ages of laboratory mice. J. Infect. Dis. 162, 133-138 (1990).

47. Wang, G. et al. Impact of genotypic variation of Borrelia burgdorferi sensu stricto on kinetics of dissemination and severity of disease in C3H/HeJ mice. Infect. Immun. 69, 4303-4312 (2001).

48. Pingen, M., Schmid, M. A., Harris, E. \& McKimmie, C. S. Mosquito Biting Modulates Skin Response to Virus Infection. Trends in Parasitology vol. 33 645-657 (2017).

49. Lestinova, T., Rohousova, I., Sima, M., de Oliveira, C. I. \& Volf, P. Insights into the sand fly saliva: Blood-feeding and immune interactions between sand flies, hosts, and Leishmania. PLoS Negl. Trop. Dis. 11, e0005600 (2017).

50. Šimo, L., Kazimirova, M., Richardson, J. \& Bonnet, S. I. The essential role of tick salivary glands and saliva in tick feeding and pathogen transmission. Front. Cell. Infect. Microbiol. 7 , $281(2017)$.

53. Teysseire, N., Chiche-Portiche, C. \& Raoult, D. Intracellular movements of Rickettsia conorii adn R. typhi based on actin polymerization. Res. Microbiol. 143, 821-829 (1992).

54. Heinzen, R. A., Hayes, S. F., Peacock, M. G. \& Hackstadt, T. Directional actin polymerization associated with spotted fever group Rickettsia infection of Vero cells. Infect. Immun. 61, 19261935 (1993).

55. Walker, D. H. The realities of biodefense vaccines against Rickettsia. Vaccine 27, D52-55 (2009). Biochemical journal. (2010). 
bioRxiv preprint doi: https://doi.org/10.1101/2020.09.23.310409; this version posted November 11,2020 . The copyright holder for this preprint (which was not certified by peer review) is the author/funder, who has granted bioRxiv a license to display the preprint in perpetuity. It is made available under aCC-BY-NC-ND 4.0 International license.

621 to lipopolysaccharide: evidence for TLR4 as the Lps gene product. J. Immunol. 162, 3749-52

622 (1999).

623 58. Müller, U. et al. Functional role of type I and type II interferons in antiviral defense. Science (80.). 264, 1918-1921 (1994).

625 59. Huang, S. et al. Immune response in mice that lack the interferon-y receptor. Science (80-. ). $626 \quad 259,1742-1745(1993)$. 


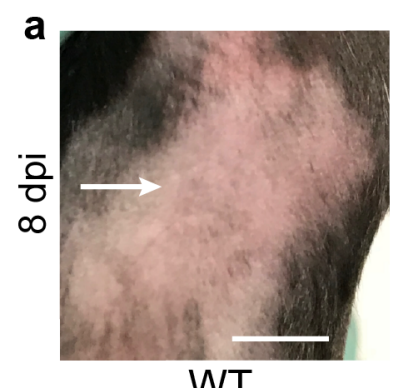

WT

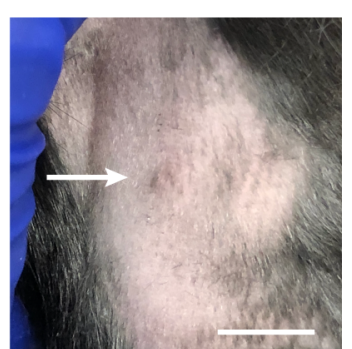

Ifnart-

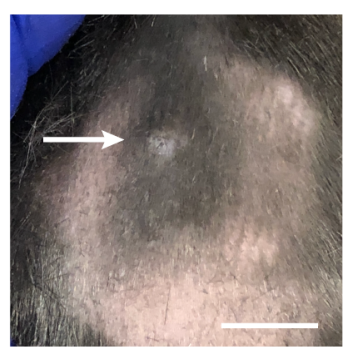

Ifngr'

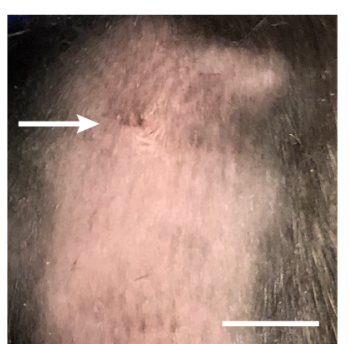

T/r4--

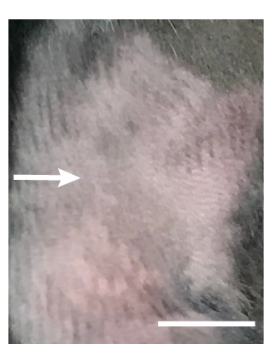

PBS

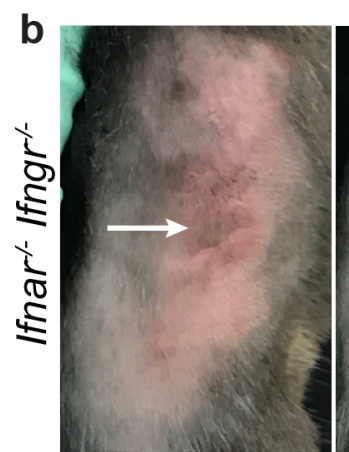

4 dpi

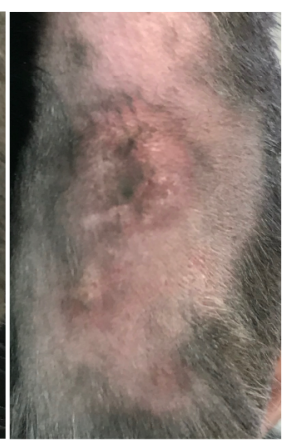

$6 \mathrm{dpi}$

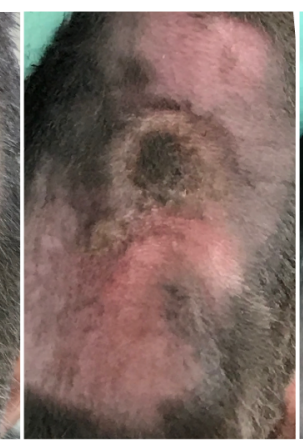

$8 \mathrm{dpi}$

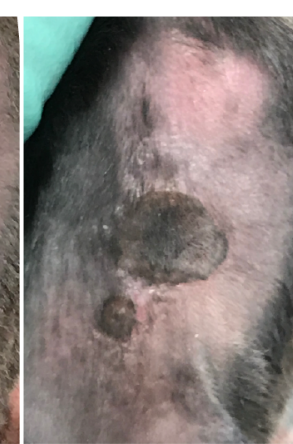

$11 \mathrm{dpi}$

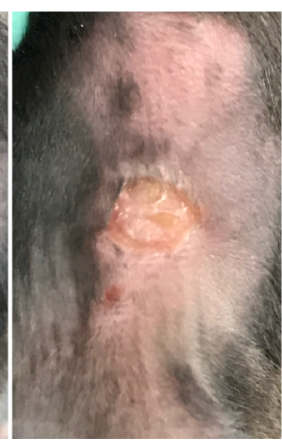

$13 \mathrm{dpi}$

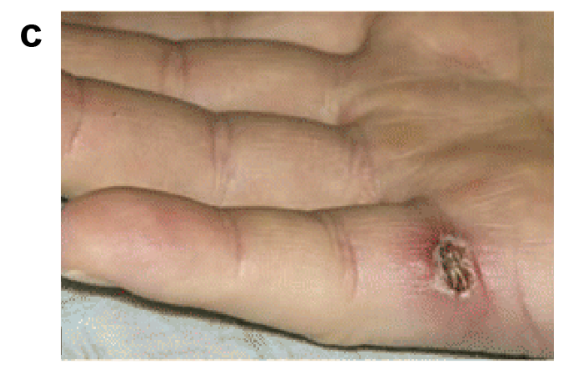

d

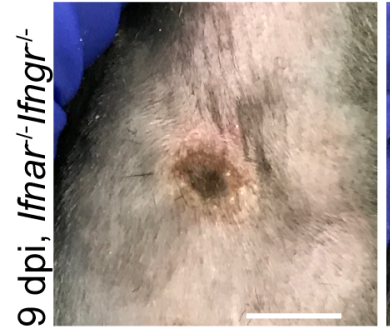

$10^{5}$ R. parkeri

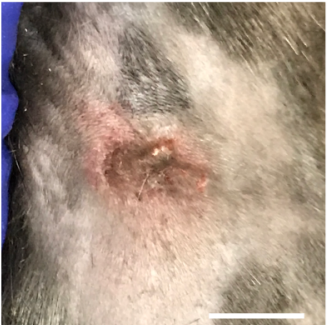

$10^{4} R$. parkeri

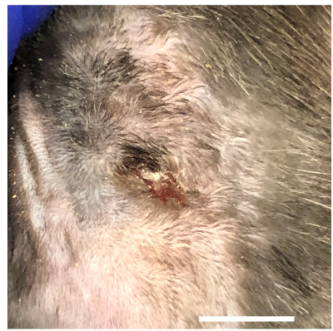

$10^{3}$ R. parkeri

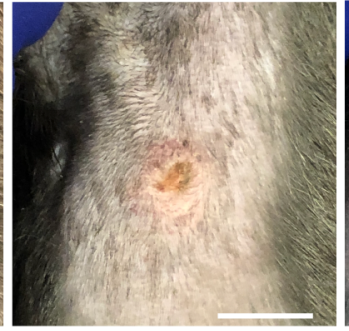

$10^{2}$ R. parkeri

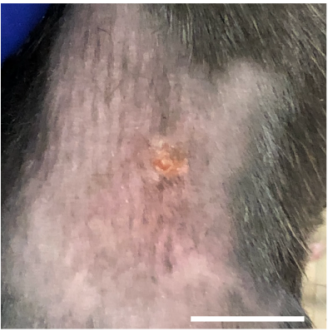

$10^{1}$ R. parkeri

Figure 1: I.d. infection of Ifnar/-Ifngr ${ }^{-/-}$mice with $R$. parkeri elicits skin lesions that are grossly similar to human eschars. a) Representative images of WT, Ifnar/ ${ }^{-/}$, Ifngr $r^{-/}$, and TIr ${ }^{-/-}$mice, infected intradermally with $10^{7}$ WT $R$. parkeri at $8 \mathrm{dpi}$ and WT mice injected with PBS. White arrows indicate the infection site on the right flank of the mouse. Scale bar, $1 \mathrm{~cm}$. Data are representative of three independent experiments. b) Representative images of an Ifnar $^{-/} /$Ifngr $^{-/-}$mouse after i.d. inoculation with $10^{7} R$. parkeri. Data are representative of 3 independent experiments. The white arrow indicates the injection site on the right flank of the mouse. Scale bar, $1 \mathrm{~cm}$. c) Gross pathology of a human $R$. parkeri infection, from Paddock et $\mathrm{al}{ }^{4}$. d) Representative images of $/$ fnar $^{-/ /} / \mathrm{fngr}^{-/-}$mice infected intradermally with the indicated amounts of WT R. parkeri at 9 d.p.i. Scale bar, $1 \mathrm{~cm}$. Data are representative of two independent experiments. 


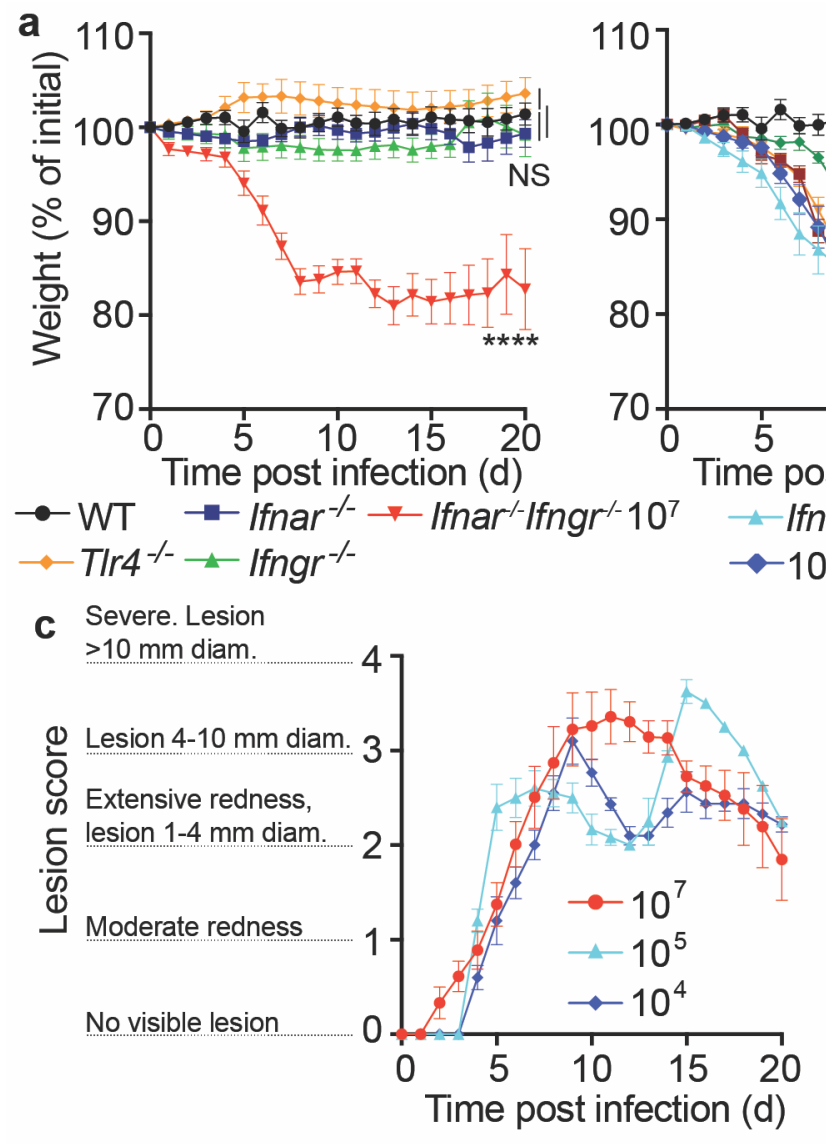

642

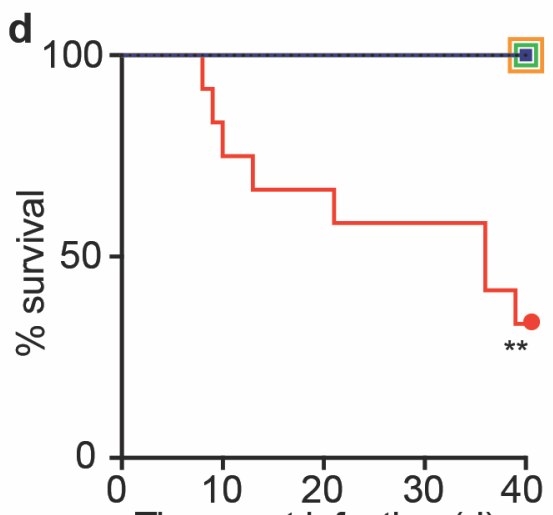

Time post infection (d)

...... WT $\square$ TIr4 ${ }^{-/}$

$\rightarrow-$ Ifnar ${ }^{\prime-} \rightarrow-$ Ifnar-/fngr/- $\left(10^{7} \mathrm{Rp}\right)$

ㅁ. Ifngr-1-

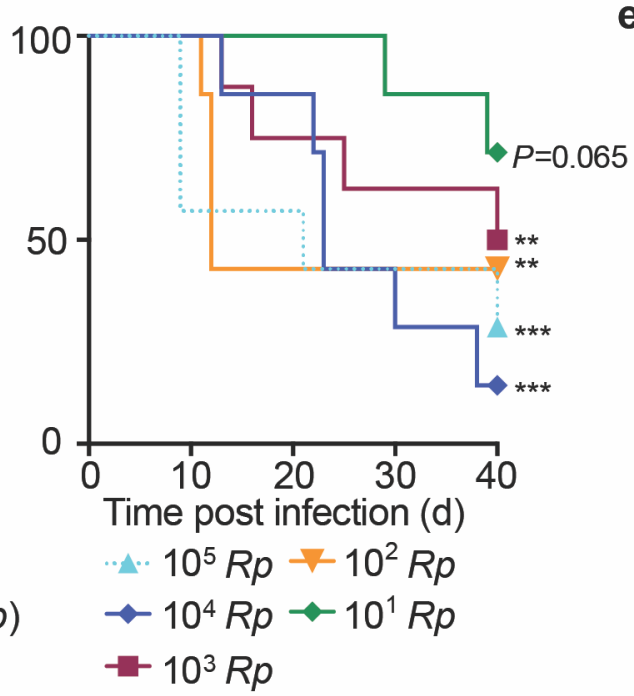

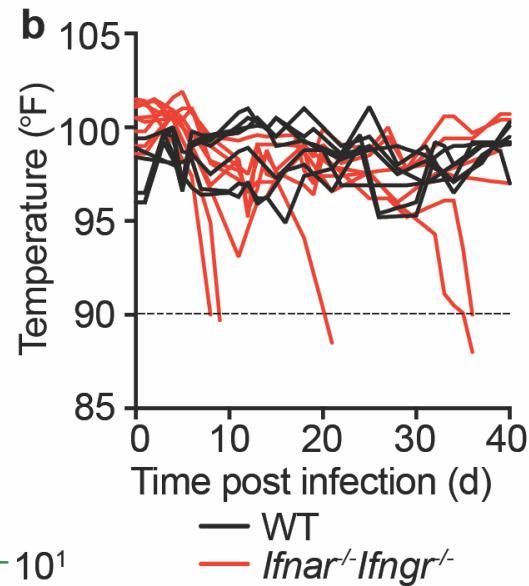

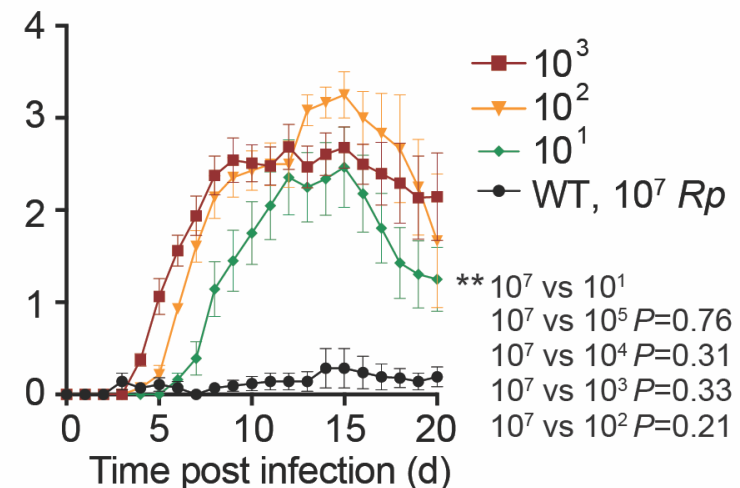

spleens livers

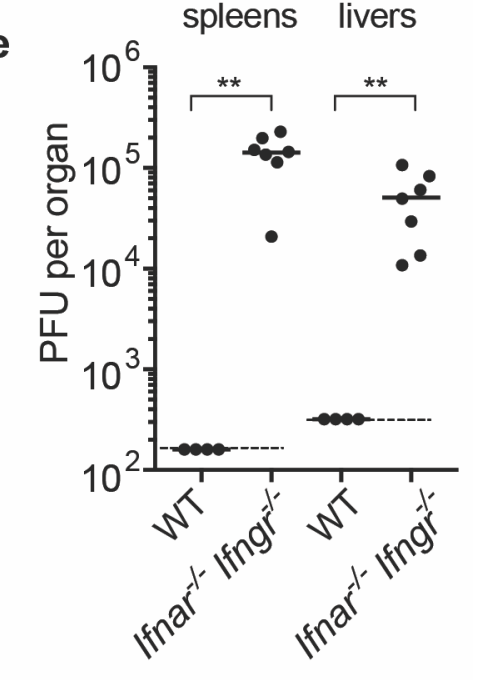

Figure 2: I.d. infection of Ifnar ${ }^{-/ / I n g r^{-/}}$mice by R. parkeri elicits disseminated, lethal disease.

a) Weight changes over time in mice infected i.d. with $R$. parkeri. Data are shown as a percentage change to initial weight. In the left panel, all mice were infected with $10^{7} R$. parkeri; $n=7$ (WT), $n=11$

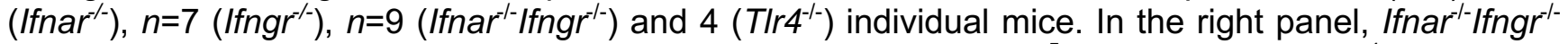
mice were infected with the indicated amounts of $R$. parkeri; $n=7\left(10^{5} R\right.$. parkeri), $n=7\left(10^{4} R\right.$. parkeri), $n=8\left(10^{3}\right.$ R. parkeri), $n=7\left(10^{2} R\right.$. parkeri), $n=7\left(10^{1} R\right.$. parkeri) individual mice. WT data is the same in both panels. Data for each genotype are combined from two or three independent experiments. b) Temperature changes over time in mice intradermally infected with $10^{7} R$. parkeri. Each line is an individual mouse. Mice were euthanized if their temperature fell below $90^{\circ} \mathrm{F}$, as indicated by the dotted line. Data are the combination of three independent experiments with $n=7$ (WT) and 9 (Ifnar $r^{-/} /$Ifngr $\left.^{-/}\right)$ individual mice. c) Analysis of gross skin pathology after i.d. infection. Ifnar ${ }^{-/} /$fngr $^{-/-}$mice were infected with the indicated number of $R$. parkeri and monitored over time. WT mice were infected with $10^{7} R$. 
parkeri. Data are the combination of three independent experiments for WT and the $10^{7}$ dose in Ifnar ${ }^{-/-}$ 656 Ifngr $r^{-/}$mice; data for all other doses are the combination of two independent experiments. $n=9\left(10^{7}\right)$, $657 n=5\left(10^{5}\right), n=5\left(10^{4}\right), n=8\left(10^{3}\right), n=7\left(10^{2}\right), n=7\left(10^{1}\right)$, and $n=7$ (WT) individual mice. d) Mouse survival 658 after i.d. infection with R. parkeri. In the left panel, all mice were infected with $10^{7}$ R. parkeri; $n=7$ (WT),

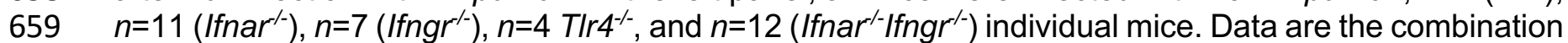
660 of three separate experiments for WT, Ifnar, and Ifnar//fngr/ and two separate experiments for Ifngr $r^{/-}$ 661 and $T / r 4^{-/}$. In the right panel, Ifnar/-Ifngr $r^{-/}$mice were infected with the indicated amounts of $R$. parkeri. 662 Data are the combination of two independent experiments; $n=7\left(10^{5}\right), n=7\left(10^{4}\right), n=8\left(10^{3}\right), n=7\left(10^{2}\right)$, 663 and $n=7\left(10^{1}\right)$ individual mice. e) Bacterial burdens in organs of intradermally infected WT and Ifnar ${ }^{-/-}$ 664 Ifngr $^{-1-}$ mice. Mice were intradermally inoculated with $10^{7} \mathrm{R}$. parkeri, and spleens and livers were 665 harvested and plated for p.f.u. at 72 h.p.i. Dotted lines indicate the limit of detection. Data are the 666 combination of two independent experiments. $n=4$ (WT) and 7 (Ifnar ${ }^{-/} /$fngr $\left.^{-/}\right)$individual mice. Data in a, 667 c are the mean \pm SEM. Statistical analyses in a used a two-way ANOVA where each group was compared to WT at $t=20$ d.p.i. Statistical analyses in c used a two-way ANOVA at $t=20$ d.p.i. Statistical analyses in $\mathbf{d}$ used a log-rank (Mantel-Cox) test to compare Ifnar $^{-/-}$to $/$fnar $^{-/} / \mathrm{fngr}^{-/-}$at each dose. Statistical analysis in e used a two-tailed Mann-Whitney $U$ test. NS, not significant; ${ }^{* *} P<0.01$; 

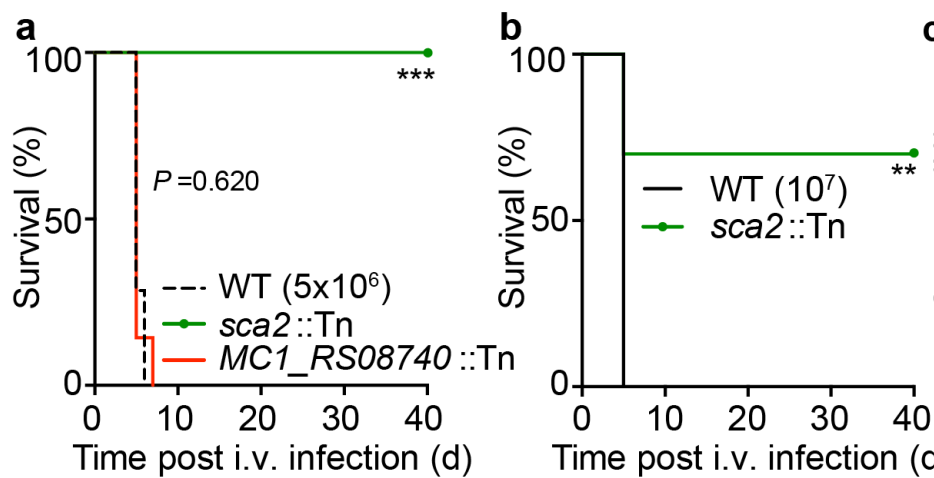

Time post i.v. infection (d)

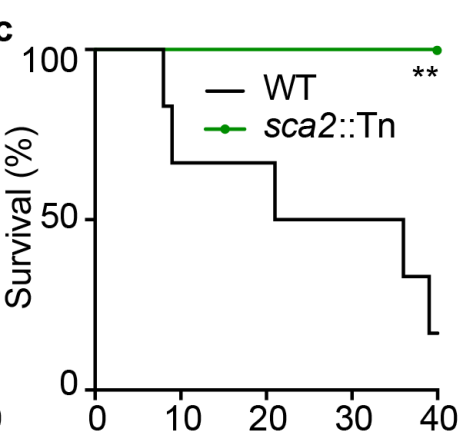

Time post i.d. infection (d)

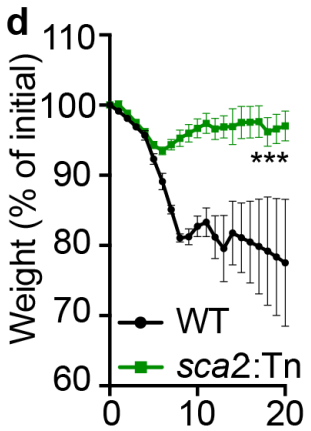

Time post i.d. (d)
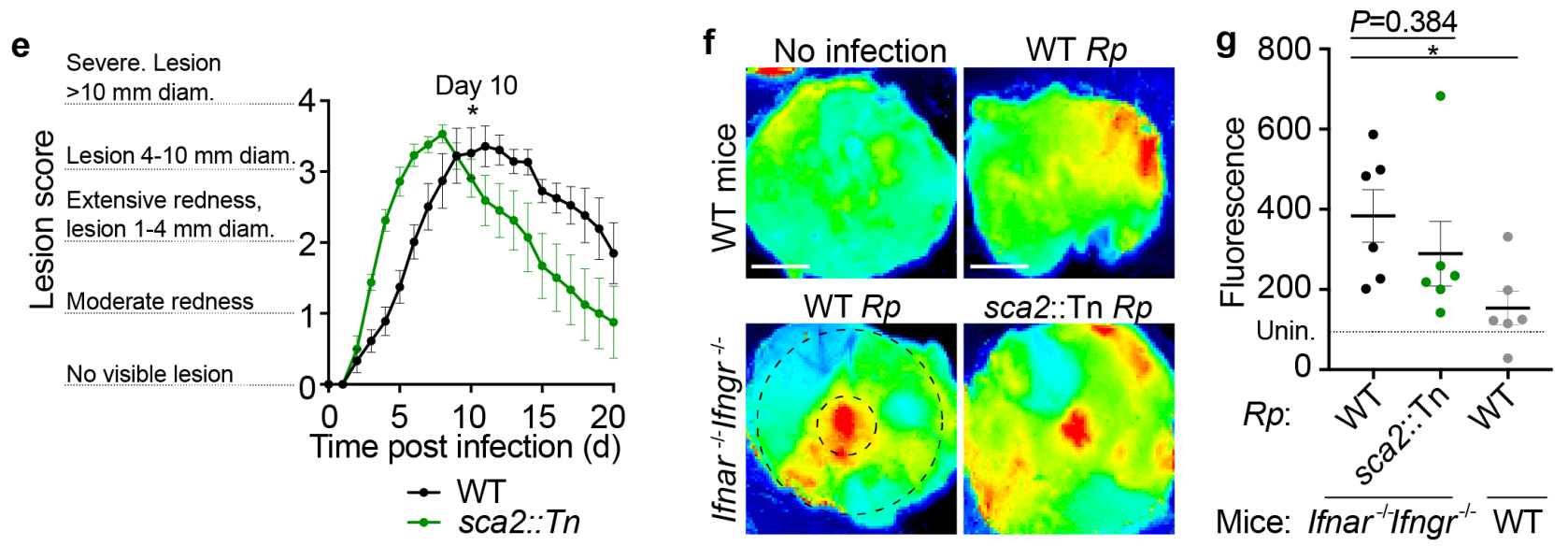

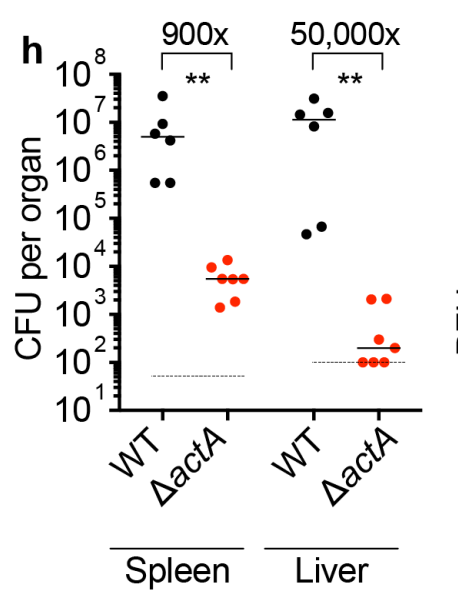

L. monocytogenes, i.v.

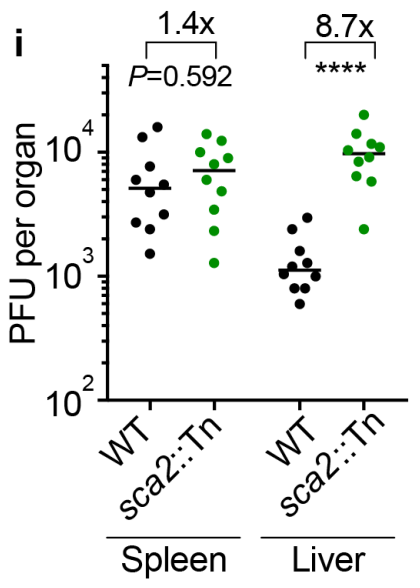

$10^{7}$ R. parkeri, i.v.

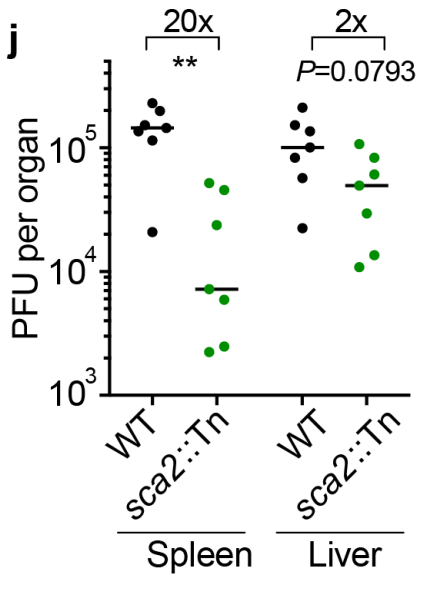

$10^{7}$ R. parkeri, i.d.

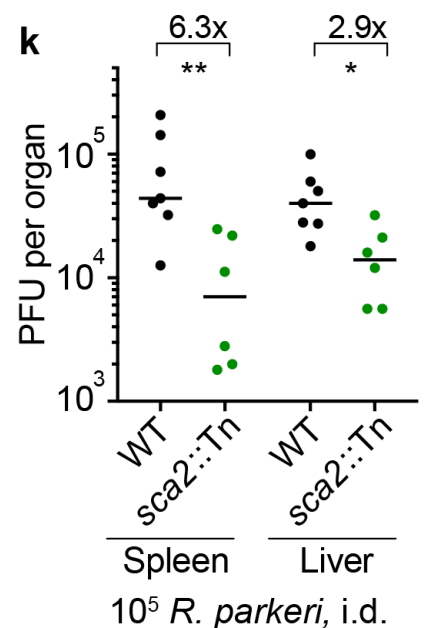

675

677

678

679

680

681

682

683

684

685

686

687

Figure 3: $R$. parkeri Sca2 contributes to dissemination from skin to spleens and livers.

a) Survival of Ifnar ${ }^{-/}$Ifngr ${ }^{-/}$mice upon i.v. infection with $5 \times 10^{6}$ R. parkeri. $n=7$ (WT), 10 (sca2::Tn), and 7 (MC1_RS08740::Tn R. parkeri) individual mice. Data are the combination of two independent experiments. b) Survival of Ifnar ${ }^{-/}$Ifngr $r^{-/-}$mice upon i.v. infection with $10^{7} R$. parkeri. $n=7$ (WT) and 10 (sca2::Tn) individual mice. Data are the combination of two independent experiments. c) Survival of Ifnar ${ }^{/-I f n g} r^{\prime-}$ mice upon i.d. infection with $10^{7}$ R. parkeri. $n=6$ (WT) and 8 (sca2::Tn) individual mice. Data are the combination of two independent experiments. d) Weight changes of Ifnar/-/fngr $r^{-/}$mice upon i.d. infection with $10^{7}$ R. parkeri. $n=6$ (WT) and 8 (sca2::Tn) individual mice. Data are the combination of two independent experiments. e) Analysis of gross skin pathology after i.d. infection. Ifnar $r^{-/} / \mathrm{fngr}^{-/}$mice were infected with $10^{7}$ of the indicated strains of $R$. parkeri and monitored over time. $n=9$ (WT) and 8 (sca2::Tn) individual mice. Data are the combination of two independent experiments. f) Representative images of fluorescence in mouse skin after i.d. infection with $10^{6} R$. parkeri and delivery of a fluorescent 
dextran, at 5 d.p.i. Scale bars, $1 \mathrm{~cm}$. The larger black dashed circle represents the area that was measured for fluorescence for each sample, as indicated in Fig. $\mathbf{3 g}(\sim 80,000$ pixels). The smaller blackdashed circle represents of the injection site area that was measured for fluorescence for each sample, as indicated in Fig. S3 ( 7,800 pixels). g) Quantification of fluorescence in mouse skin after i.d. infection. Mice were infected with $10^{7}$ R. parkeri, and $150 \mu$ fluorescent dextran was intravenously delivered at 5 d.p.i. Skin was harvested $2 \mathrm{~h}$ later, and fluorescence was measured using a fluorescence imager. Data indicate measurements of larger areas of skin, as indicated in $\mathbf{f}$ by the larger black circle. $n=6$ (WT R. parkeri) and $n=6$ (sca2::Tn R. parkeri) individual Ifnar ${ }^{-/}$Ifngr ${ }^{-/-}$mice; $n=6$ (WT R. parkeri) individual WT mice. For each experiment, the average of uninfected samples was normalized to 100; each sample was divided by the average for uninfected mice and multiplied by 100; the dotted horizontal line indicates 100 arbitrary units, corresponding to uninfected (unin.) mice. Data are the combination of two independent experiments. h) Quantification of $L$. monocytogenes abundance in organs of WT C57BL/6J mice upon i.v. infection with $10^{4}$ bacteria, at 72 h.p.i. Data are the combination of two independent experiments. $\mathrm{n}=6(\mathrm{WT}), \mathrm{n}=7$ ( $\Delta$ actA) individual mice. i) Quantification of $R$. parkeri abundance in spleens and livers of WT C57BL/6J mice upon i.v. infection, at 72 h.p.i. Data are the combination of two independent experiments. $n=10$ (WT) and 10 (Sca2::Tn) individual mice. j) Quantification of $R$. parkeri abundance in organs upon i.d. infection with $10^{7} R$. parkeri. $n=7$ (WT) and 7 (sca2::Tn) individual mice. Data are the combination of two independent experiments. Data for WT $R$. parkeri in Ifnar/-/fngr $r^{-/}$mice are the same as in Fig. 2e. k) Quantification of $R$. parkeri abundance in organs upon i.d. infection with $10^{5}$ R. parkeri. $n=7$ (WT) and 6 (sca2::Tn). Data are the combination of two independent experiments. Solid horizontal bars in $\mathbf{g}$ indicate means; solid horizontal bars in $\mathbf{h}-\mathbf{k}$ indicate medians; error bars indicate SEM. Statistical analyses for survival in a, b, c used a log-rank (Mantel-Cox) test. Statistical analysis in $\mathbf{d}$ used a two-way ANOVA at $\mathrm{t}=20$. Statistical analysis in $\mathbf{e}$ used a two-way ANOVA from 0 to 10 d.p.i. Statistical analyses in $\mathbf{g}$ used a two-tailed Student's $\mathrm{T}$ test. Statistical analyses in $\mathbf{h}, \mathbf{i}, \mathbf{j}, \mathbf{k}$ used a two-tailed Mann-Whitney $U$ test. The fold change in $\mathbf{h}, \mathbf{i}, \mathbf{j}, \mathbf{k}$ indicates differences of medians. ${ }^{*} P<0.05 ;{ }^{* *} P<0.01$; ${ }^{* *} P<0.001 ;{ }^{* * *} P<0.0001$. 


\section{a}

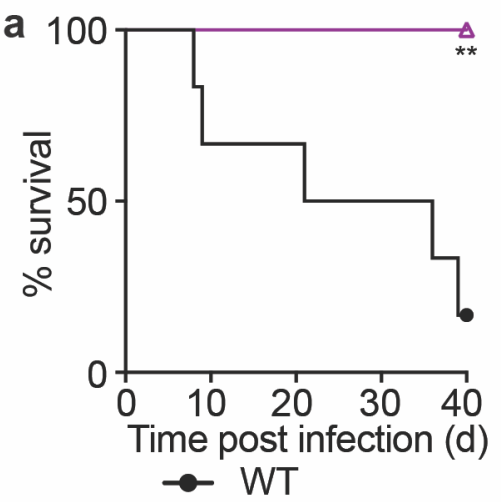

$\rightarrow$ WT

C Severe. Lesion

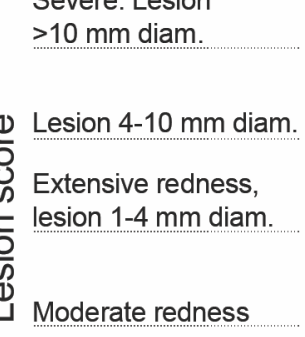

No visible lesion b

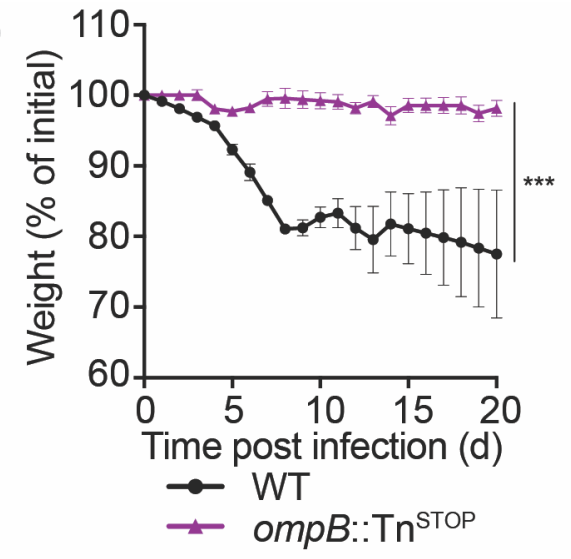

\section{Figure 4: ompB mutant $R$. parkeri elicit no lethality and reduced skin lesion formation in Ifnar $^{-{ }^{-}}$} Ifngr/- mice.

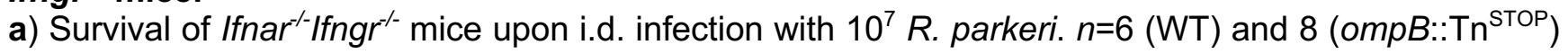
individual mice. Data are the combination of two independent experiments. Data for WT are the same as in Fig. 3c. b) Weight changes of Ifnar ${ }^{-/} / f_{n g} r^{-/}$mice upon i.d. infection with $10^{7} R$. parkeri. $n=6$ (WT) and 8 (ompB:: $\mathrm{Tn}^{\text {STOP }}$ ) individual mice. Data are the combination of two independent experiments. Data for WT are the same as in Fig. 3d. c) Analysis of gross skin pathology after i.d. infection. Ifnar-/ffngr/mice were infected with $10^{7}$ of the indicated strains of $R$. parkeri and monitored over time. $n=9$ (WT) and $8\left(\mathrm{ompB}:: \mathrm{Tn}^{\mathrm{STOP}}\right.$ ) individual mice. Data are the combination of two independent experiments. Data for WT are the same as in Fig. 3e. Error bars indicate SEM. Statistical analyses in a used a log-rank (Mantel-Cox) test. Statistical analysis in $\mathbf{b}$ used a two-way ANOVA from 0 to 20 d.p.i. Statistical analysis in c used a two-way ANOVA from 0 to 10 and 20 d.p.i. ${ }^{* *} P<0.01 ;{ }^{* * *} P<0.001$. 
a 10

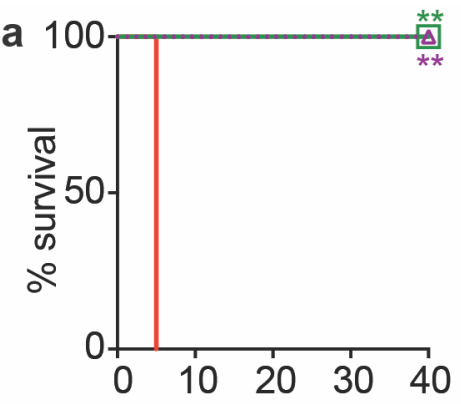

Time post infection (d)

- naïve

$$
\text { 曰 sca2::Tn immunized }
$$

. $\triangle$. ompB::Tn ${ }^{\text {STOP }}$ immunized

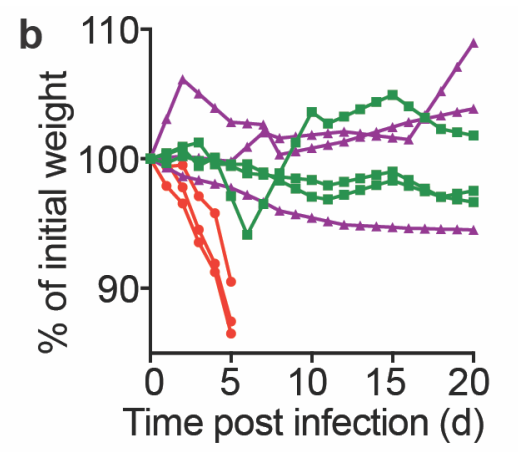

$\rightarrow$ naïve

- sca2::Tn immunized

- omp $B:: \mathrm{Tn}^{\text {STOP }}$ immunized

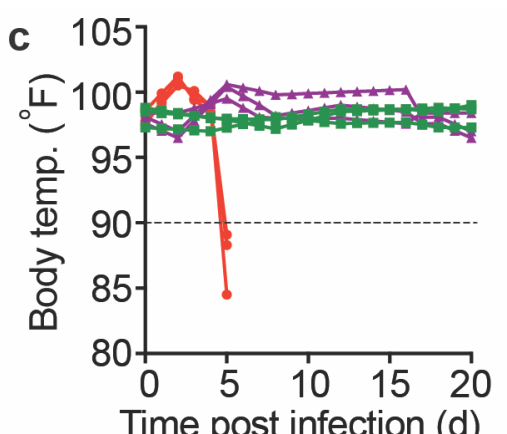

Time post infection (d)

$\rightarrow$ naïve

- sca2 ::Tn immunized

- ompB::: $\mathrm{Tn}^{\text {STOP }}$ immunized

Figure 5: ompB and sca2 mutant $R$. parkeri elicit immunity in Ifnar/-Ifngr/- mice.

a) Survival of immunized and naïve Ifnar ${ }^{-/} / \mathrm{fngr}^{-/-}$mice upon i.v. R. parkeri infection. Immunized mice were first infected with $5 \times 10^{6}$ sca2::Tn or $10^{7}$ ompB:Tn ${ }^{\text {STOP }} R$. parkeri and were re-challenged $40 \mathrm{~d}$ later with $10^{7}$ WT R. parkeri. $n=6$ (naïve); $n=5$ (sca2::Tn immunized); $n=5$ (ompB::Tn ${ }^{\text {STOP }}$ immunized) individual mice. Data are the combination of two independent experiments. b) Weight changes over time in mice infected i.d. with $10^{7} R$. parkeri. Data are representative of two independent experiments. $n=3$ (naïve); $n=3$ (sca2::Tn immunized); $n=3$ (ompB::Tn ${ }^{\text {stop }}$ immunized) individual mice. Each line represents an individual mouse. c) Temperature changes over time in mice infected i.d. with $10^{7} R$. parkeri. Data are representative from two independent experiments. $n=3$ (naïve); $n=3$ (sca2::Tn immunized); $n=3$ (ompB::Tn ${ }^{\text {STOP }}$ immunized) individual mice. Each line represents an individual mouse. Statistical analyses in a used a log-rank (Mantel-Cox) test to compare each group of immunized mice to naïve mice. ${ }^{* *} P<0.01$. 


\section{Supplemental Figures}

a

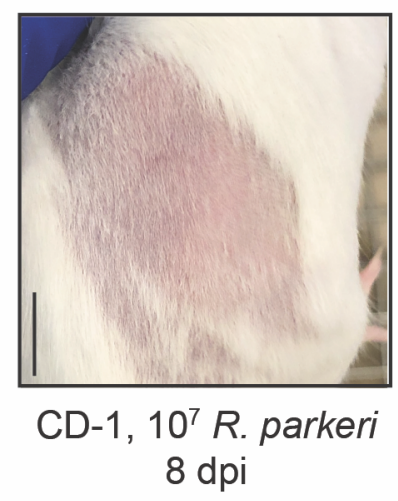

b

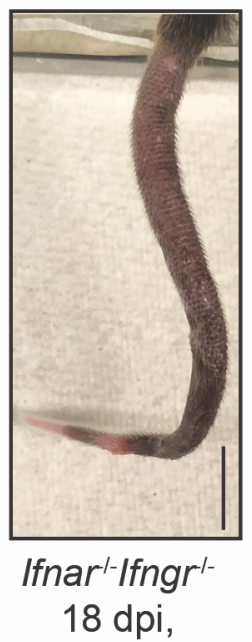

i.d.

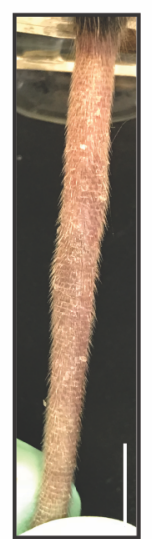

Ifngr ${ }^{\text {l- }}$ mouse \#1 34 dpi,

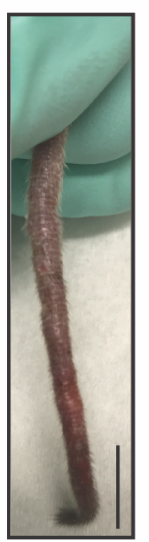

Ifngr'- mouse \#2 $9 \mathrm{dpi}$,

i.v.

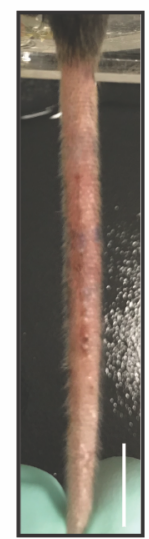

Ifngr'- mouse \#3 $14 \mathrm{dpi}$,

i.v.

Figure S1: Ifnar/-Ifngr $r^{-1-}$ mice develop disseminated disease upon intradermal $R$. parkeri infection.

a) Representative image of the right flank of CD-1 mice intradermally infected with $10^{7} R$. parkeri. Scale bar, $1 \mathrm{~cm}$. Data are representative from two independent experiments.

b) Representative images of tails of Ifnar ${ }^{-/} / f_{n g} r^{-/}$and Ifngr/- mice, infected via the i.v. or i.d. route (as indicated), with $10^{7}$ WT $R$. parkeri. Some Ifnar/-/fngr $r^{-/-}$and Ifngr $r^{-/}$mice had no gross pathological manifestations in the tail, whereas some mice exhibited inflamed, necrotic tails at various times post infection. Scale bar, $1 \mathrm{~cm}$. Data are representative from three independent experiments. 
a

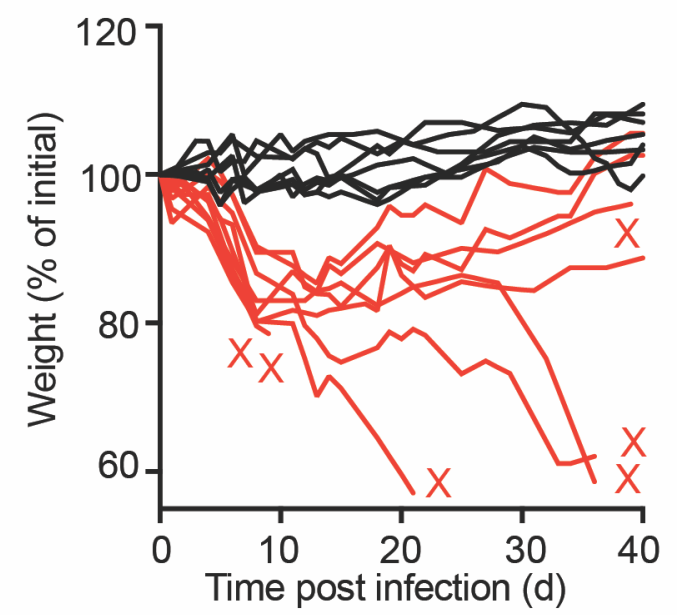

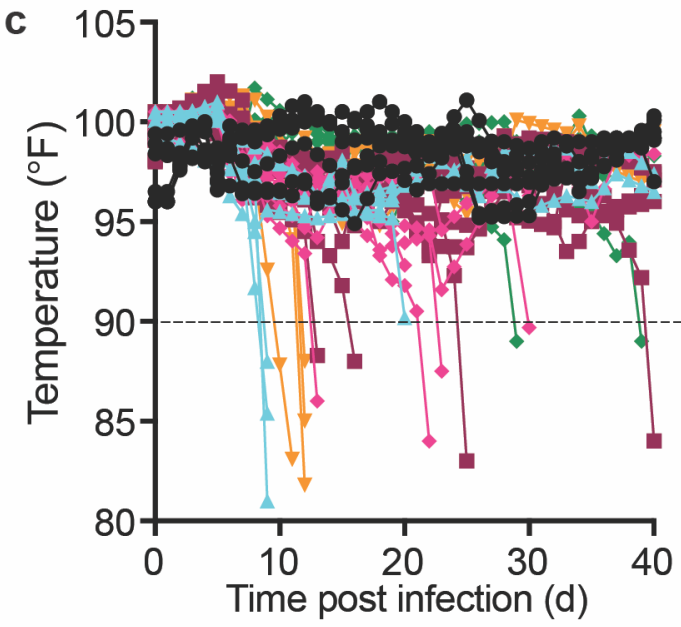

$\rightarrow$ WT
$\rightarrow$ Ifnar

b

755

Figure S2: Ifnar $r^{-/}$or Ifngr $r^{-/}$mice develop limited disease upon intradermal infection, and IfnarIfngr ${ }^{/-}$develop lesions of dose-dependent severity.

a) Weight changes over time in mice intradermally infected with $10^{7}$ WT $R$. parkeri. Data are the combination of two independent experiments for WT and three for Ifnar ${ }^{-1 /} / f_{n g r} r^{-1} ; n=7$ (WT) and $n=9$ (Ifnar ${ }^{\prime-I} / \mathrm{fngr}^{-/}$) individual mice. Each line is an individual mouse. b) Gross pathological analysis of the skin infection site after i.d. infection. Ifnar//Ifngr//-mice were infected with the indicated number of $R$. parkeri and monitored over time. Data are the combination of three independent experiments for the $10^{7}$ dose and two independent experiments for all other doses. $n=7$ (WT), $n=9\left(10^{7}\right), n=5\left(10^{5}\right), n=5\left(10^{4}\right), n=8$ $\left(10^{3}\right), n=7\left(10^{2}\right)$, and $n=7\left(10^{1}\right)$ individual mice. Data are the same as in Fig. $2 \mathrm{c}$ but are extended to 40 d.p.i. Dara are represented as means and error bars indicate SEM. c) Temperature changes over time in mice infected i.d. with the indicated amounts of WT R. parkeri. Data are the combination of two independent experiments; $n=7$ (WT), $n=7\left(10^{5}\right), n=7\left(10^{4}\right), n=8\left(10^{3}\right), n=7\left(10^{2}\right)$, and $n=7\left(10^{1}\right)$ individual mice. Each bar represents an individual mouse. Mice were euthanized if their body temperature fell below $90^{\circ} \mathrm{F}$, as indicated by the dotted line. 


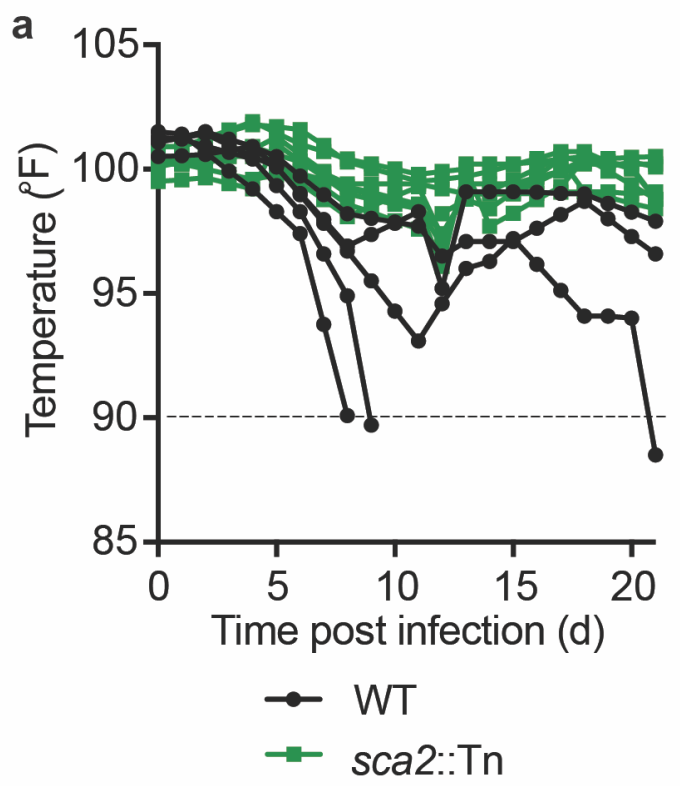

Figure S3: Intradermal infection of Ifnar ${ }^{\prime-I f n g r^{-/}}$mice with sca2::Tn $R$. parkeri causes less severe temperature loss as compared to WT bacteria.

a) Temperature changes over time in mice infected i.d. with $10^{7} R$. parkeri. Data are the combination of two independent experiments; $n=5$ (WT), $n=8$ (sca2::Tn) individual mice. Each line represents an individual mouse. Mice were euthanized if their body temperature fell below $90^{\circ} \mathrm{F}$, as indicated by the dotted line.

782 

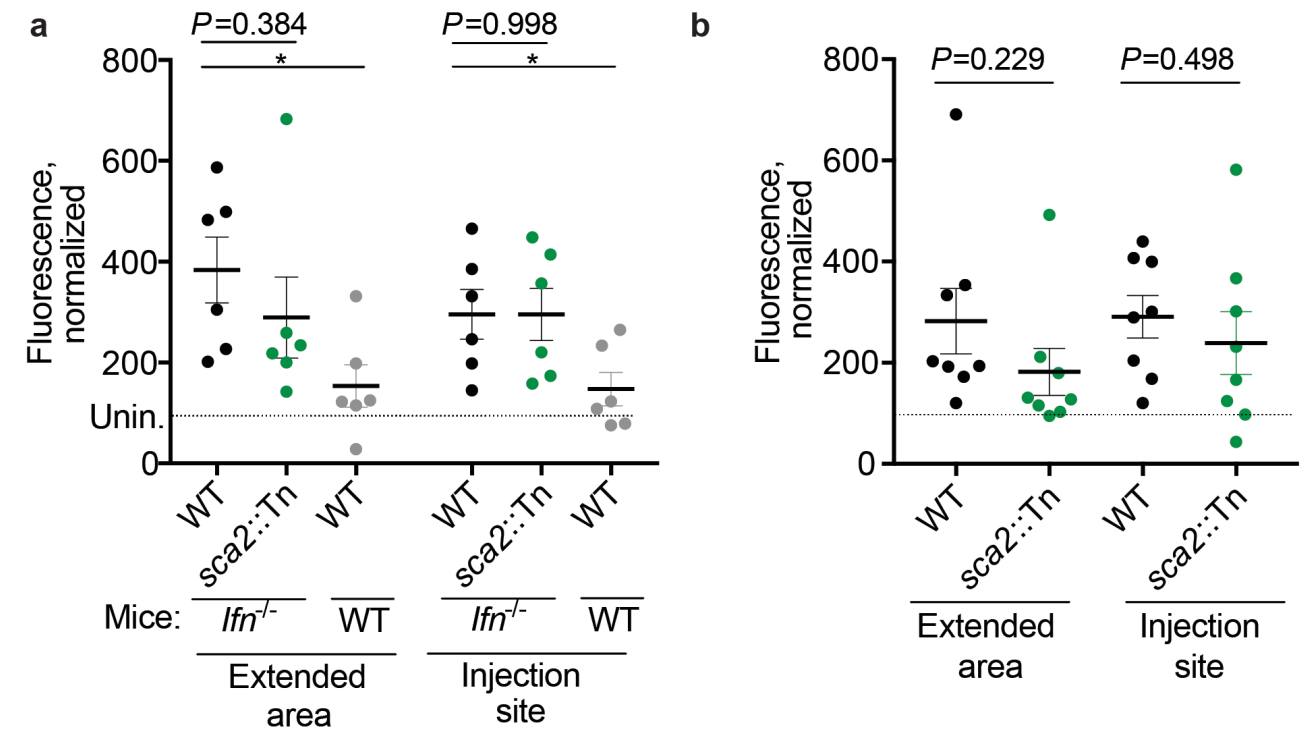

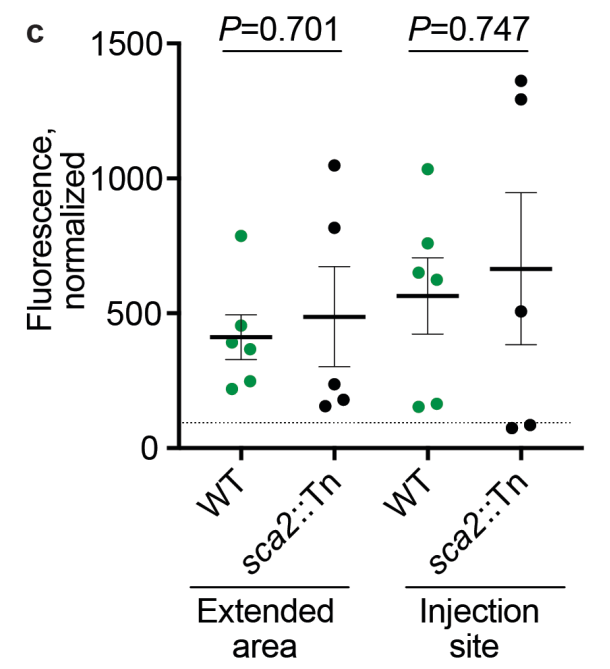

Figure S4: WT and sca2::Tn R. parkeri elicit similar amounts of vascular damage in skin upon i.d. infection of Ifnar//Ingr/- mice.

a) Quantification of fluorescence in mouse skin after i.d. infection. Mice were infected i.d. with $10^{7} R$. parkeri and fluorescent dextran was intravenously delivered at 5 d.p.i. Skin was harvested $2 \mathrm{~h}$ after delivery of dextran and analyzed with a fluorescence imager. $n=6$ (WT R. parkeri) and $n=6$ (sca2::Tn $R$. parkeri) individual Ifnar ${ }^{-/}$Ifngr ${ }^{-/-}$mice; $n=6$ (WT $R$. parkeri) individual WT mice. Data in the 'extended area' are the same as those reported in Fig. 3e. b) Quantification of fluorescence in mouse skin after i.d. infection. Mice were infected i.d. with $10^{6} \mathrm{R}$. parkeri, and fluorescent dextran was intravenously delivered at 5 d.p.i. Skin was harvested $2 \mathrm{~h}$ after delivery of dextran and analyzed with a fluorescence imager. $n=8$ (WT R. parkeri) and $n=8$ (sca2::Tn R. parkeri) individual Ifnar ${ }^{\prime-I} / f_{n} r^{\prime-}$ mice. c) Quantification of fluorescence in mouse skin after i.d. infection. Mice were infected i.d. with $10^{5} \mathrm{R}$. parkeri and fluorescent dextran was intravenously delivered at 5 d.p.i. Skin was harvested $2 \mathrm{~h}$ after delivery of dextran and analyzed with a fluorescence imager. $n=6$ (WT R. parkeri) and $n=5$ (sca2::Tn R. parkeri) individual $/ \mathrm{fnar}^{-/} / \mathrm{fngr}^{-/-}$mice. For each experiment, the average of uninfected samples was normalized to 100 , and each sample was divided by the average for uninfected mice and multiplied by 100; the dotted horizontal line indicates 100 arbitrary units, corresponding to uninfected (unin.) mice. Representative sizes for the larger 'extended areas' of skin and the smaller 'injection site' areas of skin are indicated in Fig. 3d. Data are each the combination of two independent experiments. Solid horizontal bars indicate means; error bars indicate SEM. For statistical analyses, a two-tailed Student's $\mathrm{T}$ test was used to compare the indicated samples. 


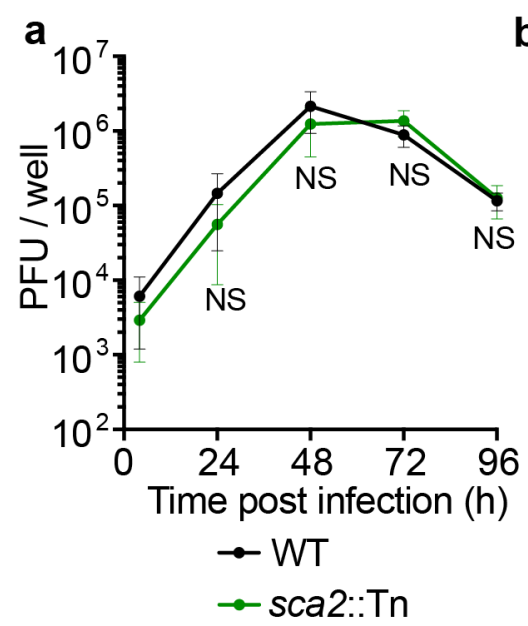

e

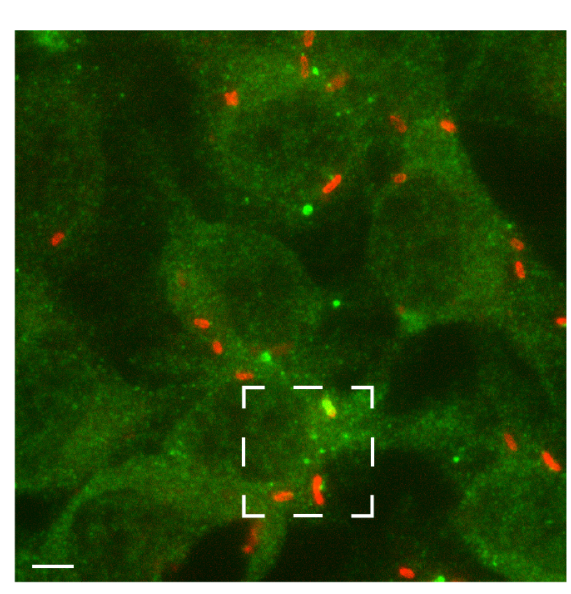

b

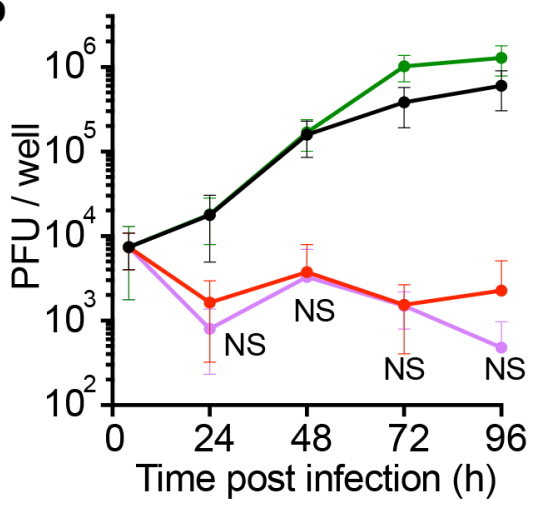

$\rightarrow$ WT $\rightarrow$ WT + IFN- $\beta$

$\rightarrow$ sca2::Th $\rightarrow$ sca2::Tn + IFN- $\beta$

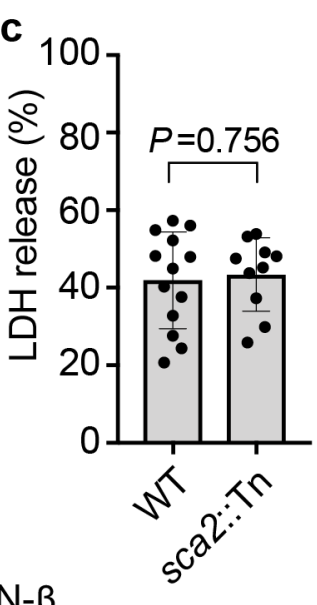

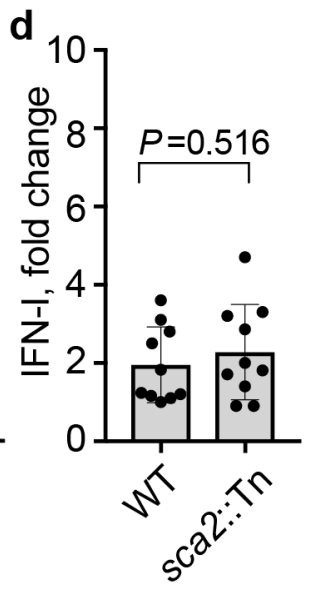
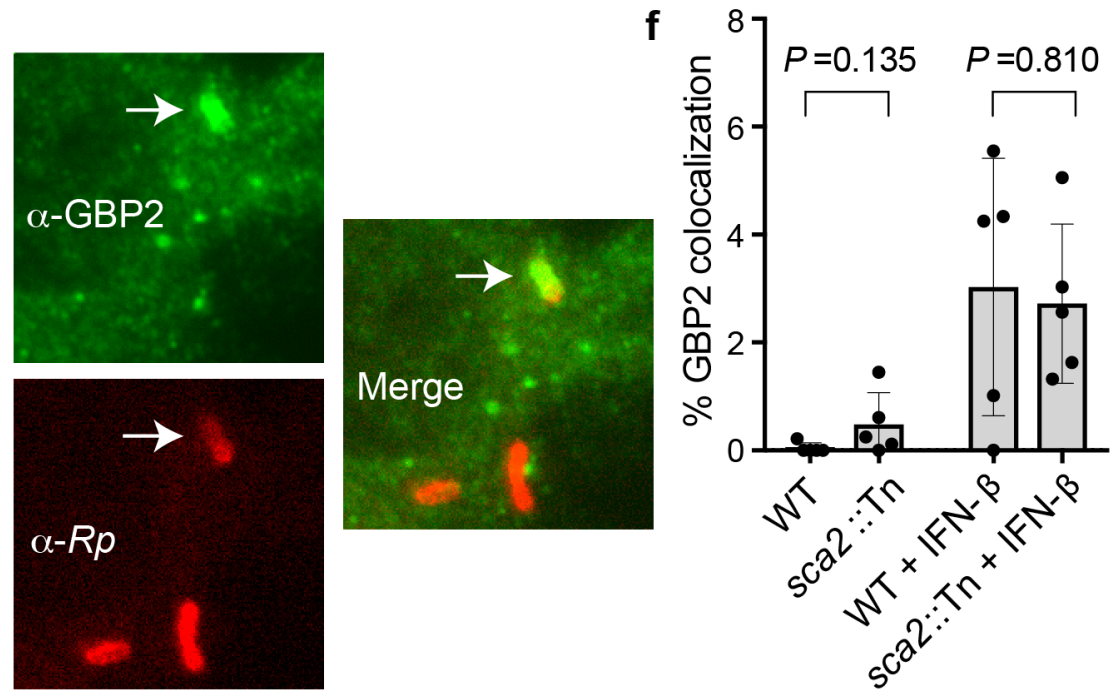

806

807

808

809

810

811

812

813

814

815

816

817

818

819

820

821

822

823

824

825

826

827

828

829

Figure S5: Sca2 does not significantly enhance $R$. parkeri avoidance of antibacterial innate immune responses in vitro.

a) $R$. parkeri abundance in HMEC-1s, multiplicity of infection (MOI) of 0.2. Data are the combination of three independent experiments, each with two biological replicates. For statistics, a two-tailed Student's T test was used to compare WT to sca2::Tn at 48, 72, and 96 h.p.i. No statistically significant differences were observed at any time. b) R. parkeri abundance in BMDMs, MOI of 1. Data are the combination of three independent experiments, each with two biological replicates. Data were normalized by multiplying fold difference between WT and Sca2::Tn at 4 h.p.i. to Sca2::Tn and Sca2::Tn + IFN-I data at all time points. c) Host cell death upon R. parkeri infection of BMDMs, as measured by lactate dehydrogenase (LDH) release assay, MOI of 1 . From left to right, $n=6$ and 3 biological replicates and are the combination of two independent experiments. d) IFN-I abundance in supernatants of infected BMDMs (24 h.p.i.; MOI of 1), measured using a luciferase reporter assay. The data show the fold change over uninfected cells. $n=7$ and 7 biological replicates and are the combination of two independent experiments. e) A representative image using $\times 100$ confocal immunofluorescence microscopy of WT BMDMs infected with sca2::Tn R. parkeri in the presence of $100 \cup$ recombinant IFN- $\beta$ (3 h.p.i.; MOI of 1). Green, $\alpha$-GBP2; red, $\alpha$-Rickettsia $(R p)$. The dotted square indicates the image that is expanded in the other images, separated into two individual and one merged channel. Scale bars, $2.5 \mu \mathrm{m}$. White arrows indicate a bacterium that colocalizes with GBP2. Data are representative of three independent experiments. f) Quantification of GBP2 colocalization with R. parkeri in BMDMs at 24 h.p.i. Each data point is an average of at least five separate images totaling $>150$ bacteria. Data are the combination of three independent experiments. Statistical analyses used a two-tailed Student's T test. NS, not significant. Data in a,b are means; bars in $\mathbf{c}, \mathbf{d}$, and $\mathbf{f}$ are means; error bars indicate SD. 